\title{
Cross-tissue transcriptome-wide association studies of 885,176 individuals and seven diseases of the gut-brain axis identify susceptibility genes shared between schizophrenia and inflammatory bowel disease
}

David Ellinghaus ( $\square$ d.ellinghaus@ikmb.uni-kiel.de)

Institute of Clinical Molecular Biology https://orcid.org/0000-0002-4332-6110

Florian Uellendahl-Werth

Christian-Albrechts-University of Kiel

Carlo Maj

Institute for Genomic Statistics and Bioinformatics

\section{Oleg Borisov}

Institute for Genomic Statistics and Bioinformatics

Simonas Juzenas

https://orcid.org/0000-0001-9293-0691

Eike Wacker

Institute of Clinical Molecular Biology https://orcid.org/0000-0002-2068-2550

Isabella Jørgensen

University of Copenhagen https://orcid.org/0000-0001-5694-4332

Tim Steiert

Institute of Clinical Molecular Biology

Saptarshi Bej

University of Rostock

The IBD Genetics Consortium

International PSC Study Group

Peter Krawitz

https://orcid.org/0000-0002-3194-8625

Per Hoffmann

Institute of Human Genetics

Christoph Schramm

First Department of Internal Medicine

\section{Olaf Wolkenhauer}

University of Rostock https://orcid.org/0000-0001-6105-2937

Karina Banasik

University of Copenhagen https://orcid.org/0000-0003-2489-2499

Søren Brunak

University of Copenhagen https://orcid.org/0000-0003-0316-5866

Stefan Schreiber

Institute for Clinical Molecular Biology and Department of General

Tom Karlsen

Oslo University Hospital

Franziska Degenhardt

University of Duisburg-Essen

Markus Nöthen

School of Medicine \& University Hospital Bonn https://orcid.org/0000-0002-8770-2464

Andre Franke

Christian-Albrechts-University Kiel

Trine Folseraas

Oslo University Hospital Rikshospitalet

Article

Keywords: Genetic Correlations, Shared Molecular Mechanisms, Enteroendocrine and Paneth-like Cells, Calcineurin-related Signaling Pathways 
DOl: https://doi.org/10.21203/rs.3.rs-590423/v1

License: (c) (i) This work is licensed under a Creative Commons Attribution 4.0 International License. Read Full License

Version of Record: A version of this preprint was published at Communications Biology on January 20th, 2022. See the published version at https://doi.org/10.1038/s42003-022-03031-6. 


\section{Abstract}

Genetic correlations and an increased incidence of psychiatric disorders in inflammatory-bowel disease (IBD) have been reported, but shared molecular mechanisms are unknown. We performed cross-tissue and multiple-gene conditioned transcriptome-wide association studies (TWAS) for 23 tissues of the gutbrain-axis (GBA) using GWAS data sets (total 246,772 patients and 638,454 controls) for Crohn's disease (CD), ulcerative colitis (UC), primary sclerosing cholangitis (PSC), schizophrenia (SCZ), bipolar disorder (BD), major depressive disorder (MDD) and attention-deficit/hyperactivity disorder (ADHD). We identified NR5A2, SATB2, and PPP3CA (encoding a target for calcineurin inhibitors in refractory UC) as shared susceptibility genes with transcriptome-wide significance both for CD/UC and SCZ, largely explaining fine-mapped association signals at nearby GWAS susceptibility loci. Analysis of bulk and single-cell RNA-sequencing data showed that PPP3CA expression was strongest in neurons and in enteroendocrine and Paneth-like cells of the ileum, colon, and rectum, indicating a possible link to the GBA. Gene-disease associations as part of calcineurin-related signaling pathways such as NFAT activation or Wnt are the most likely shared candidate pathways for IBD and SCZ, where risk is mediated by gene expression.

\section{Introduction}

The direct link (gut-brain-axis [GBA]) of intestinal dysfunction and inflammation with brain development and mental illness is one of the most current topics in biomedical research ${ }^{1-3}$. Gastrointestinal symptoms have been observed in patients with neurobehavioral, neurodevelopmental and mental diseases, including attention-deficit/hyperactivity disorder (ADHD) ${ }^{4}$, schizophrenia $(\mathrm{SCZ})^{5}$ and major depressive disorder (MDD) ${ }^{6}$. Vice versa, psychiatric comorbidity, including depression, anxiety, bipolar disorder (BD) and SCZ, is known to be more prevalent in inflammatory bowel disease (IBD) patients ${ }^{7-9}$. Given the polygenic nature of most psychiatric and chronic inflammatory diseases, some of the (potentially shared) phenotypic variation in disease risk is expected to be explained by expression quantitative trait loci (eQTL) active in disease-relevant tissues of the GBA ${ }^{10}$.

In this study, we performed cross-tissue transcriptome-wide association studies (TWAS) for 23 tissues of the GBA using GWAS summary statistics from ten case-control GWAS data sets (total 246,772 patients and 638,454 controls, Supplementary Table 1; Methods) of three clinically related inflammatory diseases of the gastrointestinal tract (CD, UC and primary sclerosing cholangitis (PSC) because PSC patients suffer from a highly increased frequency (62-83\%) of IBD called PSC-IBD $\left.{ }^{11}\right)$ and four diseases of the mind and brain (SCZ, MDD, BD and ADHD) for which moderate to strong genetic correlation $\left(r_{\mathrm{g}}>0.3\right)$ at the genome-wide level has been described between chronic inflammatory diseases ${ }^{12}$ and psychiatric diseases ${ }^{13}$, respectively. The TWAS approach can be viewed as a transcriptome-wide screening method to test for gene-disease associations from GWAS data sets ${ }^{14}$. By using a new principled cross-tissue TWAS method $\left(\right.$ UTMOST) ${ }^{15}$ - simultaneously training expression imputation models across multiple disease-relevant tissues and performing cross-tissue gene-level conditioned association tests - in combination with GWAS/TWAS conditional analysis for cross-phenotype studies we were able to address several types of confounding factors that have previously compromised the validity of TWAS studies for complex diseases ${ }^{14,16}$ : (1) poor TWAS prediction accuracy due to imputation from reference expression panels containing tissues not relevant to disease, (2) a bias in the number of transcriptome-wide significant genedisease associations due to different sample sizes of reference datasets in expression imputation models ${ }^{17}$, (3) an excess of TWAS association signals at loci with multiple TWAS signals due to correlated expression among genes of the same locus and (4) an inaccurate determination of association boundaries for TWAS signals based on SNP information.

The objectives of this cross-tissue, cross-phenotype TWAS were to (i) evaluate the effectiveness of cross-tissue imputation analysis using ten GWAS consortium studies of psychiatric and inflammatory disease; (ii) identify transcriptome-wide significant gene-disease associations in GBA tissues for each disease and localize the most strongly associated genes through cross-tissue and multiple-gene-conditioned fine-mapping analyses; (iii) quantify the overall component of (shared) genetically regulated expressional changes $\left(\rho_{\mathrm{E}}\right)$ for immune-mediated and psychiatric diseases in relation to genome-wide genetic correlation $\left(\rho_{\mathrm{G}}\right)$; (iv) identify comorbidities and causal relationships using population-based administrative health data from the entire Danish population and by performing Mendelian Randomization (MR) analyses; (v) identify susceptibility genes and pathways shared between psychiatric disorders and inflammatory diseases of the gastrointestinal tract using bulk RNA-Seq data of different developmental stages and single-cell RNA-sequencing (scRNA-seq) expression data for tissues of the GBA.

\section{Results}

\section{Cross-tissue transcriptome-wide association studies (TWAS) for seven diseases of the GBA}

The UTMOST framework was used to perform cross-tissue TWAS for 17,290 genes and 23 selected disease-relevant tissues, which had been identified as disease relevant for diseases of the GBA in a previous study of Finucane and colleagues ${ }^{18}$ (Methods), using transcriptome-wide and genome-wide reference data of 4,043 samples provided by consortia projects GTEx ${ }^{19}$, STARNET ${ }^{20}$, and BLUEPRINT ${ }^{21}$ (Fig. 1, Supplementary Table 2). A schematic overview of the study workflow is shown in Supplementary Fig. 1. Gene expression imputation was conducted using GWAS summary statistics data of ten consortium metaanalysis studies comprising 246,772 independent cases of European-ancestry (CD: 21,771; UC: 18,621; PSC: 4,338; SCZ: 36,989; MDD: 135,458; BD: 9,412; ADHD: 20,183 ) and 638,454 healthy controls (Supplementary Table 1). We then tested for gene-disease associations for a maximum of 17,290 genes and 23 tissues for each of the ten GWAS meta-analysis studies. Single-tissue gene-wise (marginal; i.e. unconditioned) association $P$ values (analysis Singletissue $_{\text {marginal; }}$ Supplementary Table 3) were combined into (marginal) joint-tissue gene-wise summary $P$ values (analysis GBJ $J_{\text {marginal, }}$ resulting in one $P_{\text {GBJ_marginal }}$ for each gene and disease; Supplementary Table 3) via a generalized Berk-Jones (GBJ) test ${ }^{22}$. The cross-tissue GBJ gene test quantifies genedisease associations across all tissues of the GBA (separately for each disease), adjusts the correlation between TWAS association statistics for individual tissues, and also provides (compared with standard univariate meta-analysis approaches) a substantial increase in statistical power in situations where eQTL 
effects are not unique to a single tissue (Methods). The GBJ test identified a total of 586 (277), 345 (179), 214 (79), 361 (104), 38 (21), 77 (10) and 42 (8) gene-disease associations (number of loci with size of $\pm 1 \mathrm{Mb})$ with transcriptome-wide significance $\left(P_{\mathrm{GBJ} \_ \text {marginal }}<0.05 / 15,587=3.20 \times 10^{-6}\right.$; Supplementary Fig. 2; corrected for a maximum of 15,587 successfully tested and GBJ-meta-analyzed genes) for CD, UC, PSC, SCZ, MDD, BD, ADHD, respectively (Supplementary Table 3). To evaluate a possible bias in the number of transcriptome-wide significant gene-disease associations due to different sample sizes of reference data sets, we further conducted TWAS analyses using pre-trained expression imputation models provided by S-PrediXcan ${ }^{23}$ and FUSION ${ }^{24}$ (Methods). We observed on average only a moderate positive correlation between reference sample size and the number of significant genes per tissue in our cross-tissue TWAS results (average Spearman's $\rho_{\text {UTMOST }}=0.46$; Supplementary Table 4, Supplementary Fig. 3 ). This suggests that cross-tissue TWAS imputation models can effectively select predictive cis $\mathrm{QQTL}$ variants (within $\pm 1 \mathrm{Mb}$ of the transcription start/end site of a gene) shared by multiple tissues, whereas tissue-specific eQTLs with strong effects were retained.

\section{Cross-tissue and multiple-gene-conditioned fine-mapping analyses at loci with multiple gene-disease association signals}

$P_{\text {GBJ_marginal }}$ values from cross-tissue TWAS analyses (Supplementary Table 3 ) showed weak to moderate inflation of GBJ association statistics $\left(\lambda_{1000}\right.$ in the range [0.94;1.05]; Supplementary Table 5). One reason for this may be co-regulation of multiple genes by the same eQTL, as well as LD between eQTLs with non-zero prediction weights, which may lead to correlated predicted expression between nearby genes and thus multiple correlated TWAS hits per locus ${ }^{14}$, analogous to the situation in a GWAS study where an association signal typically spans a large number of highly significant SNPs in high LD with the causal $\mathrm{SNP}^{25}$. In order to distinguish between causal and marker gene-disease associations at significant TWAS loci, we performed (i) multiple-gene-conditioned

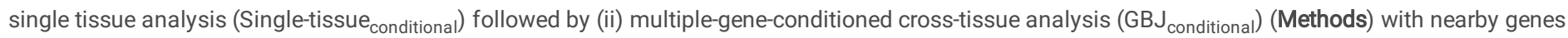
within range of $\pm 1 \mathrm{Mb}$ around transcriptome-wide significant genes from marginal results (genes highlighted as red dots in the Manhattan plots in Supplementary Fig. 2). This accounted for potential cross-tissue co-regulation and LD between eQTL SNPs, and furthermore, the marginal association result of each gene was conditioned based on all genes within $\pm 1 \mathrm{Mb}$ region (avoiding specification of fixed locus boundaries based on LD blocks from genotype data). This analysis yielded a total of 215 (80), 152 (50), 150 (22), 89 (53), 9 (5), 5 (5), 10 (6) GBJ gene-disease associations (separate loci of $\pm 1 \mathrm{Mb})$ with transcriptome-wide significance $\left(P_{\mathrm{GBJ}}\right.$ conditional $<3.20 \times 10^{-6}$ ) for $\mathrm{CD}, \mathrm{UC}, \mathrm{PSC}, \mathrm{SCZ}, \mathrm{MDD}, \mathrm{BD}, \mathrm{ADHD}$, respectively (Supplementary Table 3 and Supplementary Table 6, Methods), thus representing final transcriptome-wide significant cross-tissue multiple-gene-conditioned TWAS gene-disease association results (Table 1, Fig. 2a) within and outside the boundaries of established GWAS susceptibility loci (Supplementary Table 7).

Table 1

Transcriptome-wide significant $\left(P_{\text {conditional }}<3.20 \times 10^{-6}\right)$ genes from multiple-gene-conditioned fine-mapping analysis at loci with multiple gene-disease association signals, for psychiatric and immune phenotypes in 23 gut-brain-axis (GBA) tissues. For selection of 23 tissues, see Fig. 1, Supplementary Table 2

and Methods. 164 (78), 109 (62), 51 (85), 52 (56), 2 (7), 7 (7), 3 (8) TWAS susceptibility genes are within (outside) the boundaries of established GWAS susceptibility loci (Methods, Supplementary Table 7) for CD, UC, PSC, SCZ, MDD, BD, ADHD, respectively. Approximately half of all gene-disease associations are found in at least two tissues of the GBA (for more details see Supplementary Table 6).

\begin{tabular}{|c|c|c|c|c|c|c|c|}
\hline & CD & UC & PSC & SCZ & MDD & BD & ADHD \\
\hline \multirow[t]{2}{*}{ TWAS discovery $n_{\text {cases }} / n_{\text {controls }}$} & $18,431 /$ & $14,191 /$ & $3.377 /$ & $36,989 /$ & $135,458 /$ & $9,412 /$ & $20,183 /$ \\
\hline & 33,658 & 33,658 & 33,658 & 113,075 & 344,901 & 127,760 & 35,191 \\
\hline $\mathrm{n}$ unique genes [loci] across 23 tissues & $215[80]$ & $152[50]$ & $150[22]$ & 89 [53] & $9[5]$ & $5[5]$ & $10[6]$ \\
\hline $\mathrm{n}$ unique genes in total from single tissues & $242(240)$ & $171(163)$ & $136(61)$ & $108(85)$ & $9(5)$ & $14(14)$ & $11(11)$ \\
\hline n unique genes GWAS loci & $164(164)$ & 109 (109) & $51(50)$ & $52(49)$ & $2(2)$ & $7(7)$ & $3(3)$ \\
\hline n unique genes non-GWAS loci & $78(76)$ & $62(54)$ & $85(11)$ & $56(36)$ & $7(3)$ & $7(7)$ & $8(8)$ \\
\hline $\mathrm{n}$ unique genes cross-tissue* & $132(132)$ & $89(88)$ & $81(41)$ & $56(42)$ & $4(3)$ & $3(3)$ & $5(5)$ \\
\hline $\begin{array}{l}\mathrm{n} \text { unique genes cross-tissue } \\
\text { brain and non-brain }\end{array}$ & 99 (99) & $67(67)$ & $57(32)$ & $38(28)$ & $3(2)$ & $2(2)$ & $4(4)$ \\
\hline \multicolumn{8}{|c|}{ 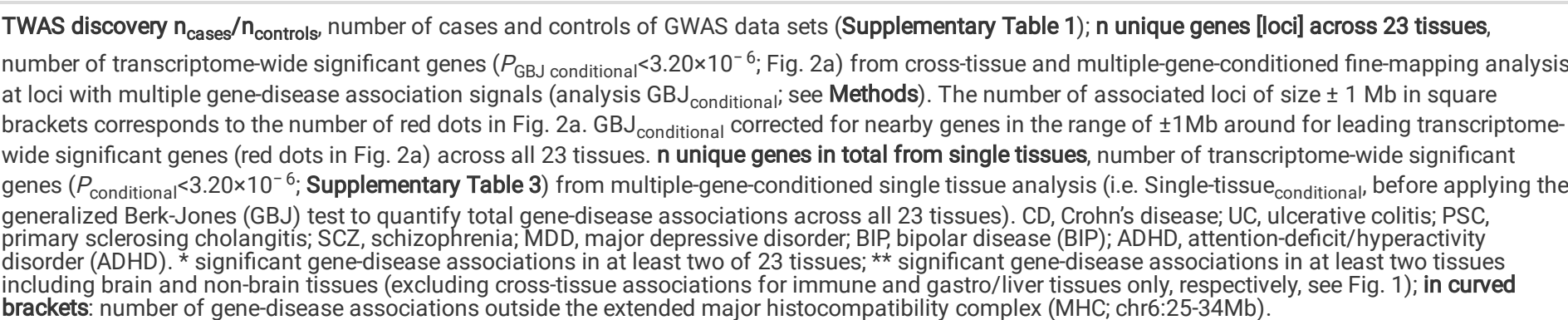 } \\
\hline
\end{tabular}

\section{Comorbidity analysis and trait correlation at the level of predicted gene expressions}

Using population-based administrative health records from the Danish National Patient Registry (DNPR), we conducted a retrospective cohort study for the period 1994 to 2018 by examining directed diagnosis pairs of 7,191,385 people from the entire Danish population (Methods). We confirmed statistically 
significant comorbidity (false discovery $P_{\mathrm{FDR}}<0.05$ ) between our four psychiatric and three immunological phenotypes for 9 psychiatric-immunological pairs out of 42 disease pairs (Supplementary Table 8) with an increased incidence of SCZ (relative risk RR $=1.15), B P(R R=1.28)$ and depression $(R R=1.52)$ in $C D$, depression in UC ( $R R=1.34)$ and PSC ( $R R=1.42), C D$ in SCZ ( $R R=1.12), B D(R R=1.29)$ and depression $(R R=1.57), U C(R R=1.37)$ and $P S C(R R=1.57)$ in depression.

Next, to examine pairwise trait correlations at the level of predicted (genetically regulated) gene expression $\left(\rho_{\mathrm{E}}\right)$ and to compare $\rho_{\mathrm{E}}$ with genome-wide genetic correlations $\left(\rho_{\mathrm{G}}\right)$, we calculated Spearman's correlations between TWAS gene effect estimates for all pairs of diseases by using the gene-wise $Z$ scores from the analyses Single-tissue marginal and $\mathrm{GBJ}_{\text {marginal }}$ and a correlation-pruned list of 1,825 co-regulation- and eQTL-independent genes previously used in Mendelian randomization studies ${ }^{26}$ (to avoid measuring correlated predicted expression on the transcriptome-wide scale) (Methods). On the single tissue level (Single-tissue ${ }_{\text {marginal }}$ S Supplementary Table 9), the strongest positive correlation across psychiatric and immune phenotypes was observed for CD ${ }^{+}$T-cells $\left(\hat{\rho}_{\mathrm{E}}\right.$ $=0.11$ for $C D$ and BP and $\hat{\rho}_{\mathrm{E}}=0.14$ for CD and SCZ) (Supplementary Fig. 4). On the cross-tissue level across all 23 tissues (GBJ marginal; Supplementary

Table 9), the strongest positive correlations across psychiatric and immune phenotypes were observed for pairs UC and BP $\left(\hat{\rho}_{\mathrm{E}}=0.15\right), \mathrm{CD}$ and $\mathrm{BP}\left(\hat{\rho}_{\mathrm{E}}=0.14\right)$, UC and SCZ ( $\left.\hat{\rho}_{\mathrm{E}}=0.13\right)$, and CD and SCZ ( $\left.\hat{\rho}_{\mathrm{E}}=0.11\right)($ Fig. $2 \mathrm{~b})$, which may explain part of the genome-wide genetic correlations $\left(\hat{\rho}_{\mathrm{G}}\right)$ for pairs UC and BP $\left(\hat{\rho}_{\mathrm{G}}=\right.$ $0.23), \mathrm{CD}$ and $\mathrm{BP}\left(\hat{\rho}_{\mathrm{G}}=0.22\right), \mathrm{UC}$ and SCZ $\left(\hat{\rho}_{\mathrm{G}}=0.14\right)$, and $\mathrm{CD}$ and SCZ ( $\hat{\rho}_{\mathrm{G}}=0.12$ ) reported by Tylee et al. ${ }^{27}$, suggesting that the effects of genetic variants shared between psychiatric and immune phenotypes are largely mediated by gene expression.

\section{Mendelian randomization analysis for psychiatric and immune phenotypes}

Mendelian Randomization (MR) is a commonly used method to assess a causal influence of one trait (exposure) on another (outcome). To identify putative pairs of immune-mediated and psychiatric diseases that may be related in a causal manner (i.e. vertical pleiotropy ${ }^{28}$ ), we conducted MR analysis for transcriptome-wide significant lead genes (i.e. our instruments with $P_{\text {conditional }}<3.20 \times 10^{-6}$ and filtered for the most-significant genes from Singletissue $_{\text {conditional }}$ within $\pm 1 \mathrm{Mb}$ regions) using multi-gene TWAS MR analysis (including HEIDI-outlier filtering that removes instruments with strong putative horizontal pleiotropic effects, where one gene has independent effects on multiple traits) as implemented in GSMR ${ }^{29}$ (Methods). After Bonferroni correction for 171 pairwise tests $\left(P_{\mathrm{GSMR}}<2.92 \times 10^{-4}\right)$, we identified $\mathrm{CD}\left(P_{\mathrm{GSMR}}=9.58 \times 10^{-5} ; \mathrm{OR}=1.10,95 \% \mathrm{Cl} 1.05-1.15\right)$ and UC $\left(P_{\mathrm{GSMR}}=8.40 \times 10^{-6} ; \mathrm{OR}=1.17,95 \% \mathrm{Cl}\right.$ $1.09-1.26)$ as a putative risk factor (exposure) for SCZ in tissues "Colon_Transverse" and "Esophagus_Gastroesophageal_Junction", respectively (Supplementary Table 10). HEIDI-filtering identified CSNK1A1 and SGSM3 as potential horizontally pleiotropic genes, suggesting a pleiotropic function in both diseases.

\section{Identification of gene-disease associations shared between psychiatric and immune phenotypes}

After identifying pairs of immune-mediated and psychiatric diseases as putative correlated traits on the transcriptome-wide level, we sought to identify susceptibility genes shared between psychiatric and immune phenotypes from the list of all transcriptome-wide significant genes from both analyses Singletissue $_{\text {conditional }}$ and $\mathrm{GBJ}_{\text {conditional }}$ (Table 1). Out of 288 possible pair-wise tissue-specific combinations of psychiatric and immune phenotypes (Supplementary Table 11), gene overlap analysis revealed 31 (of those $4 \mathrm{GBJ}_{\text {conditional) }}$ pairs of psychiatric and immune phenotypes that share at least one susceptibility gene. After excluding genes within the major histocompatibility complex (MHC; chr6:25-34Mb), which was particularly relevant to pairs PSC-MDD and PSC-SCZ in various tissues, we identified three non-MHC TWAS susceptibility genes (NR5A2, SATB2, PPP3CA) from analyses Single-tissue ${ }_{\text {conditional }}$ to be shared between brain (SCZ,CD,UC: Hypothalamus; SCZ,UC: Frontal cortex BA9; SCZ,CD: Putamen basal ganglia) and intestinal tissues (CD,UC: Colon transversum; UC: Colon sigmoideum; CD: Colon transversum) (Table 2; more detailed results in Supplementary Table 3). In summary, these three genes met the transcriptome-wide significance threshold of $3.20 \times 10^{-6}$ for SCZ and CD/UC in the same individual tissues and also showed associations across both brain and intestinal tissues, with substantial gene expression heritability estimates $h_{\text {med }}{ }^{2}$ (i.e. heritability mediated by the cis genetic component of gene expression levels) across the 23

tissues $\left(h_{\text {med-min }}{ }^{2}, h_{\text {med-max }}{ }^{2}\right)$ of $(13.4 \%, 18.2 \%)$ for NR5A2, $(6.9 \%, 24.8 \%)$ for SATB2, and $(5.0 \%, 10.9 \%)$ for PPP3CA (Table 2; Methods). Thus, these genes met the most stringent criteria for shared susceptibility genes, as they were shared between psychiatric and immune phenotypes in the same tissues and across brain and non-brain tissues and were therefore prioritized below. We observed that an increase (decrease) in predicted expression for $N R 5 A 2$ and SATB2 (PPP3CA) was associated with an increased risk of SCZ, CD and/or UC (Table 2). In addition, four other gene candidates (INO80E, SF3B1, SGSM3, $Z C 3 H 7 B)$ from the secondary analysis ( $\left.G B J_{\text {conditional }}\right)$ met the transcriptome-wide significance threshold and showed associations across both brain and intestinal tissues, but each in different tissues for psychiatric and immune phenotypes, implying that for these four genes the most significant tissues differ between psychiatric and immune phenotypes (Supplementary Tables 11-13). This suggests that those four genes may be involved in SCZ and CD/UC, but not necessarily in the same tissue. We will show below by gene set enrichment analysis that INO8OE, SGSM3, ZC3H7B are likely to be proxies for other genedisease associations at the respective loci. 
Table 2

Transcriptome-wide significant susceptibility genes (NR5A2, SATB2, PPP3CA) shared between schizophrenia and inflammatory bowel diseases expressed in . tissues. Gene overlap analysis of TWAS results from multiple-gene-conditioned fine-mapping analysis (Single-tissue conditional; Supplementary Table 11, wi

Supplementary Table 3) identified three common TWAS susceptibility genes (NR5A2, PPP3CA, SATB2) that meet the transcriptome-wide significance thresholı for each disease separately; for diseases listed in the "Diseases primary tissue(s)" column). These three genes are shared between psychiatric and immune $p$ tissues and across brain and non-brain tissues (Fig. 3). Increased predicted expression (indicated by a positive Z score) of NR5A2 and SATB2 is associated wit $\mathrm{CD}$ as well as SCZ and UC. Decreased predicted expression (indicated by a negative $Z$ score) of PPP3CA is associated with increased risk for SCZ and CD. Ge major histocompatibility complex (MHC; chr6:25-34Mb) were excluded from gene overlap analysis.

\begin{tabular}{|c|c|c|c|c|c|c|c|c|c|c|c|c|}
\hline \multirow[t]{2}{*}{ Gene } & \multirow[t]{2}{*}{$\begin{array}{l}\text { Gene-startend } \\
\text { (in kb) }\end{array}$} & \multirow{2}{*}{$\begin{array}{l}\left(h_{\text {med-min }}{ }^{2},\right. \\
\left.h_{\text {med-max }}{ }^{2}\right) \\
\text { in \% }\end{array}$} & \multirow{2}{*}{$\begin{array}{l}\text { Primary } \\
\text { tissue(s) of } \\
\text { strongest } \\
\text { gene-disease } \\
\text { association }\end{array}$} & \multirow{2}{*}{$\begin{array}{l}\text { Diseases } \\
\text { primary } \\
\text { tissue(s) }\end{array}$} & \multirow{2}{*}{$\begin{array}{l}\text { Secondary } \\
\text { tissue(s) of } \\
\text { gene- } \\
\text { disease } \\
\text { association }\end{array}$} & \multicolumn{3}{|c|}{$\begin{array}{l}Z_{\text {marginal }} \text { primary } \\
\text { tissue }\end{array}$} & \multicolumn{3}{|c|}{$P_{\text {conditional }}$ primary tissue } & \multirow{2}{*}{$\begin{array}{l}0 \\
W \\
G \\
\text { lc }\end{array}$} \\
\hline & & & & & & SCZ & $C D$ & UC & SCZ & CD & UC & \\
\hline \multirow[t]{2}{*}{ NR5A2 } & \multirow[t]{2}{*}{$\begin{array}{l}\text { chr1:199,996 } \\
-200,146\end{array}$} & \multirow[t]{2}{*}{$\begin{array}{l}(13.4 \\
18.2)\end{array}$} & \multirow[t]{2}{*}{ Hypothalamus } & \multirow[t]{2}{*}{$\begin{array}{l}\text { SCZ, CD, } \\
\text { UC }\end{array}$} & $\begin{array}{l}\text { SCZ:-: } \\
\text { CD/UC: }\end{array}$ & 4.86 & 5.82 & 7.7 & $\frac{1.63 \times 10^{-}}{6}$ & $\begin{array}{l}3.66 \times 10^{-} \\
8\end{array}$ & $\begin{array}{l}1.97 \times 10^{-} \\
13\end{array}$ & $\begin{array}{l}\mathrm{N} \\
\mathrm{O}^{\prime}\end{array}$ \\
\hline & & & & & $\begin{array}{l}\text { Colon } \\
\text { transversum }\end{array}$ & & & & & & & \\
\hline \multirow[t]{2}{*}{ SATB2 } & \multirow[t]{2}{*}{$\begin{array}{l}\text { chr2:200,134- } \\
200,320\end{array}$} & \multirow[t]{2}{*}{$(6.9,24.8)$} & \multirow[t]{2}{*}{$\begin{array}{l}\text { Frontal Cortex } \\
\text { BA9 }\end{array}$} & \multirow[t]{2}{*}{$\mathrm{SCZ}, \mathrm{UC}$} & SCZ: -; UC: & 4.75 & -0.55 & 8.46 & \multirow{2}{*}{$\begin{array}{l}1.29 \times 10^{-} \\
6\end{array}$} & \multirow[t]{2}{*}{0.908} & \multirow{2}{*}{$\begin{array}{l}2.27 \times 10^{-} \\
13\end{array}$} & \multirow[t]{2}{*}{$\begin{array}{l}\mathrm{N} \\
\mathrm{O}^{\prime}\end{array}$} \\
\hline & & & & & $\begin{array}{l}\text { Colon } \\
\text { sigmoideum }\end{array}$ & & & & & & & \\
\hline \multirow[t]{2}{*}{ PPPЗСA } & \multirow[t]{2}{*}{$\begin{array}{l}\text { chr4:101,947 } \\
-102,267\end{array}$} & \multirow[t]{2}{*}{$(5.0,10.9)$} & \multirow[t]{2}{*}{$\begin{array}{l}\text { Putamen } \\
\text { basal ganglia }\end{array}$} & \multirow[t]{2}{*}{$S C Z, C D$} & SCZ: - CD: & -4.87 & -6.79 & -2.96 & \multirow{2}{*}{${ }_{6}^{1.57 \times 10^{-}}$} & \multirow{2}{*}{$\begin{array}{l}1.28 \times 10^{-} \\
10\end{array}$} & \multirow{2}{*}{$\begin{array}{l}5.54 \times 10^{-} \\
3\end{array}$} & $N$ \\
\hline & & & & & $\begin{array}{l}\text { Colon } \\
\text { transversum }\end{array}$ & & & & & & & \\
\hline
\end{tabular}

Gene, susceptibility gene shared between psychiatric and immune disease(s); Gene-startend, transcription start-end positions (including UTRs) in kilobases ( GRCh37/hg19); ( $\left.\mathrm{h}_{\text {med-min }}{ }^{2}, \mathrm{~h}_{\text {med-max }}{ }^{2}\right)$, estimated minimum/maximum gene expression heritability in percentage attributable to SNPs in the vicinity of each ! averaged across 23 different tissues (Fig. 1); Primary tissue(s), primary tissue(s) identified from multiple-gene-conditioned fine-mapping analysis (Single-tissı in which the transcriptome-wide significant gene-disease association signal occurred; Diseases primary tissue(s), diseases showing a transcriptome-wide ass

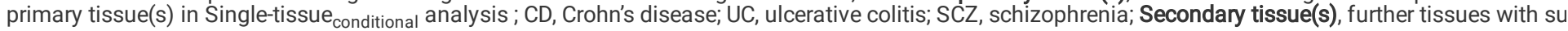
$\left(P_{\text {conditional }}<1 \times 10^{-4}\right)$ for gene-disease association; $Z$ statistic, $Z$ score from analysis Single-tissue conditional $_{\text {and/or }} \mathrm{GBJ} \mathrm{J}_{\text {conditional }}$ for primary tissue(s), the plus, increased/decreased predicted expression of these genes to be associated with increased disease risk, $Z$ scores in bold type meet the transcriptome-wide sigi $3.20 \times 10^{-6}$, TWAS $P_{\text {conditional, }} P$-value from multiple-gene-conditioned fine-mapping analyses Single-tissue conditional $_{\text {and/or }}$ GBJ $J_{\text {conditional }}$ for primary tissue(s), meet the transcriptome-wide significance threshold of $3.20 \times 10^{-6}$; Overlap with GWAS locus, testing an overlap with locus boundaries of established GWAS Ic mapping GWAS studies for $S C Z^{30}$ and $C D / U C^{31,32}$. Distance to GWAS locus, closest GWAS loci from literature for $S C Z^{30}, C D$ and $U C^{32}$ within $\pm 1 M b$ region a from column "Gene-start-end" (in kb; genome build GRCh37/hg19): SCZ: chr1:200,250 - 200,422 (1q32.1) with candidate genes LINC00862, ZNF281. CD/UC: 201,092 (1q32.1) with candidate genes CAMSAP2, RPL34P6, MRO3HP3, KIF21B, GPR25, CACNA1S, C1orf106, ASCL5. UC: chr2:199,447 - 199,709 (2q33.1). ミ 200,633 (2q33.1) with candidate genes FTCDNL 1, LOC101927641. SCZ: chr2:200,536 - 201,310 (2q33.1) with candidate genes C20rf47, C2orf69, FTCDNL 1, chr4:102,547 - 103,389 (4q24) with candidate genes BANK1, SLC39A8. CD: chr4:102,651 - 103,144 (4q24) with candidate gene BANK1.

\section{GWAS/TWAS conditional analysis to describe the GWAS contribution to the TWAS result}

To estimate the contribution of the three shared "same-tissue" susceptibility genes NR5A2, SATB2 and PPP3CA relative to the established GWAS signals as defined by the recent consortia fine-mapping studies for $\mathrm{SCZ}^{30}$ and $\mathrm{CD} / \mathrm{UC}^{31,32}$, we performed a joint GWAS/TWAS fine-mapping analysis across the 23 tissues of the GBA using the GWAS consortium summary statistics listed in Supplementary Table 1. For NR5A2, SATB2 and PPP3CA, respectively, we performed a multi-SNP-based conditional \& joint association analysis using GTCA-COJO ${ }^{33}$ to condition GWAS summary statistics on independent eQTL effects of the three genes (Methods). We examined how much of the GWAS fine-mapping signal remained after the signal of the TWAS gene eQTLs was removed. We demonstrated the applicability of our approach by examining and replicating established eQTL associations of GWAS signals as reported in the fine-mapping GWAS studies for CD/UC (Supplementary Figs. 5 and 6; detailed results showing all genes from the regions around NR5A2, SATB2, PPP3CA, INO8OE SF3B1, SGSM3, ZC3H7B and all significant tissues are described in the Supplementary Results and Supplementary_File_1.pdf). Joint GWAS/TWAS fine-mapping results for the additional gene candidates (INO8OE, SF3B1, SGSM3, ZC3H7B) from analyses GBJ conditional are discussed in Supplementary Figs. 7-10 and Supplementary Box 2.

Increased genetically regulated expression of NR5A2 in the hypothalamus and colon transversum was associated with increased risk for SCZ, CD and UC. We found that the TWAS association of NR5A2 at 1q32.1 in the hypothalamus and colon transversum (Fig. 3a) was driven by the nearby (but non-overlapping) established SCZ and CD/UC GWAS signals located 104 and 654 kilobases (kb) upstream of NR5A2, respectively. This GWAS locus was not assigned a gene in the CD/UC consortium fine-mapping study ${ }^{32}$ but was assigned an overlap with an epigenetic mark for immune cells (Immune_H3K4me1). Given the functional evidence for NR5A2 in attenuating inflammatory damage in murine and human intestinal organoids ${ }^{34}$ (see also Supplementary Box 1) we propose NR5A2 as the best candidate gene for the GWAS locus $1 \mathrm{q} 32.1$ with a possible effect on the hypothalamic-pituitary-adrenal axis (HPA axis) ${ }^{10}$.

Increased genetically regulated expression of SATB2 in frontal cortex BA9 and colon sigmoideum was associated with increased risk of SCZ and UC. The TWAS association of SATB2 at 2q33.1 (Fig. 3b) is driven by the nearby (non-overlapping) established SCZ and UC GWAS signals located 67 and 425 kilobases (kb) downstream and upstream of SATB2, respectively. Because multiple TWAS eQTLs were identified for SATB2 in the $95 \%$ credible set of the UC GWAS peak (consisting of intergenic variants between LOC101927619 and SATB2 ${ }^{32}$ ), it is convincing that the GWAS/TWAS fine-mapping analysis revealed a possible explanation of the GWAS signal by eQTLs at this locus. SATB2 as a potential susceptibility gene for UC and SCZ has already been implicated by nongenetic studies (Supplementary Box 1). 
Decreased genetically regulated expression of PPP3CA in the putamen basal ganglia and colon transversum was associated with increased risk of SCZ and CD. We found that the TWAS association of PPP3CA at 4q24 (Fig. 3c) is driven by the nearby (non-overlapping) established SCZ and CD GWAS signals located 280 and 384 kilobases $(\mathrm{kb})$ upstream of PPP3CA, respectively. GWAS fine-mapping analysis ${ }^{32}$ assigned the $95 \%$ credible set of fine-mapped SNP variants ( $\mathrm{n}=170$ SNPS) to the gene BANK1, which is closest to the lead GWAS SNP variant at $4 \mathrm{q} 24$. Because PPP3CA encodes a subunit of calcineurin (Supplementary Box 1) that has been associated with $\mathrm{SCZ}$ and is also a known drug target (due to a direct physical interaction with FKBP Prolyl Isomerase $1 \mathrm{~A}$ $(F K B P 1 A)^{35}$, also known as FKBP12) for the treatment of UC via calcineurin inhibitors, we propose PPP3CA as the best candidate gene for the GWAS association signal at $4 \mathrm{q} 24$.

\section{Analysis of bulk RNA-Seq data of different developmental stages and scRNA-seq expression data for tissues of the GBA}

Having tested the three gene-disease associations for plausibility at the GWAS level in the previous section, we hypothesized that high, tissue- or cell-typespecific expression would be indicative of a particular function in the tissue of interest, and that expression of a gene could also occur only in a small subset of cells of only a particular tissue or at a particular human developmental stage, which is hypothesized for SCZ ${ }^{36}$. We used publicly available non-diseased bulk tissue $\mathrm{e}^{37,38}$ and scRNA-Seq data sets ${ }^{39,40}$ (Methods) to look up in which tissues and at which developmental stage of human tissues and cells the shared susceptibility genes are generally expressed, and found that the observed patterns matched the existing knowledge on the genes (Supplementary Box 1 ). Using GTEx and BLUEPRINT data sets representing organ- and blood cell type-specific bulk gene expression data (Fig. 4a), we found that NR5A2 was expressed in gastrointestinal tissues but not in the brain and barely in blood cells, whereas SATB2 was expressed mainly in the colon transversum and prefrontal cortex, the same tissues where we detected the shared TWAS associations, and PPP3CA in various brain tissues, but less so in intestinal tissues. It is also possible that an adverse event in utero can interfere with normal brain development and create a brain vulnerability that, in an individual already at risk (genetic predisposition), can lead to SCZ later in life ${ }^{36}$. We therefore examined the expression of our three candidate genes in developmental data from brain ${ }^{37}$ and intestine ${ }^{38}$, since it is conceivable that a susceptibility gene is expressed during fetal development but is no longer expressed in adulthood. Again, NR5A2 was almost absent in bulk brain samples from different developmental stages, whereas SATB2 was strongly upregulated specifically in the fetal forebrain during mid-fetal development, and PPP3CA was weakly expressed in all developmental stages of the brain ${ }^{37}$ (Fig. $4 \mathrm{~b}$ ). In the intestine, it was the opposite: NR5A2 was strongly expressed in the ileum and colon during the fetal and juvenile stages, whereas SATB2 was weakly expressed in the colon sigmoideum and not ileum, and PPP3CA almost not at all ${ }^{38}$ (Fig. 4b). To achieve resolution at the single cell level, we analyzed human single-nucleus and scRNA-seq data from different cell types of the adult brain ${ }^{39}$ and gastrointestinal tract ${ }^{40}$, respectively (Fig. $4 \mathrm{c}$ ). In these data sets, NR5A2 is expressed in the ileal progenitor, stem, and transient amplifying (TA) cells, less strongly in the colon and rectum, and not in the brain, consistent with the bulk expression data. Consistent with the bulk expression from developmental stages of the intestine (Fig. 4b), SATB2 was strongly expressed in cell types of the colon and rectum and barely expressed in brain cells. PPP3CA expression was highly enriched in enteroendocrine and Paneth-like cells of the ileum, colon and rectum despite weak expression in the bulk RNA-Seq data of ileum or colon, which may imply a specific role of PPP3CA in immune modulation in the gut for CD and UC; in the brain, PPP3CA is also expressed in neurons (Fig. 4c). Bulk tissue and scRNA-seq results for genes INO8OE SF3B1, SGSM3, ZC3H7B are shown in Supplementary Fig. 11.

\section{Calcineurin-dependent NFAT and Wnt signaling as shared signaling pathways for IBD and SCZ}

Based on the statistically unambiguous gene prioritization from multiple-gene-conditioned fine-mapping analyses, it is likely that NR5A2, SATB2 and PPP3CA are shared susceptibility genes for both IBD and SCZ. The genes INO80E, SGSM3 and ZC3H7B from the secondary and not so stringent gene overlap analysis $\left(\mathrm{GBJ}_{\text {conditional }}\right)$ might be proxies for other genes with strongly correlating gene expression at the same locus (except for SF3B1 (Supplementary Figs. 7 and 11 ), where the gene-disease association signal of the eleven nearby genes of SF3B1 clearly disappeared after conditioning, see Supplementary_File_1.pdf), because these three loci showed an extremely high gene density with 45 (INO80E), 16 (SGSM3) and 29 (ZC3H7B) genes (Supplementary_File_1.pdf) making the multiple-gene-conditioned fine-mapping analysis difficult ${ }^{14}$. To test whether any of the secondary gene candidates could be linked to NR5A2, SATB2 and PPP3CA, we performed gene set enrichment analysis as implemented in Enrich $\mathrm{R}^{41}$ using the NCATS BioPlanet pathway resource of 1,658 curated human pathways ${ }^{42}$ and 27 input genes including our seven candidate genes (NR5A2, SATB2, PPP3CA, SF3B1, INO8OE, SGSM3 and ZC3H7B; Supplementary Table 12), 19 correlated transcriptome-wide significant candidate genes after multiple-gene conditioned analysis for INO8OE, SGSM3 and ZC3H7B, and CSNK1A1 (a pleiotropic HEIDI gene outlier for SCZ and UC from MR analysis (Supplementary Table 10) representing a case of horizontal pleiotropy). The strongest associated term was "calcineurin-dependent NFAT signaling role in lymphocytes" (enrichment $P=8.57 \times 10^{-7}$; Supplementary Table 14) including genes CSNK1A1, EP300 (same locus as SGSM3) and MAPK3 (same locus as INO80E) in addition to our main candidate gene PPP3CA. In our view, EP300 and MAPK3 are the more likely candidates instead of SGSM3 and INO8OE, respectively. Both genes are important signal transducers and are known to be part of or intersect with the Wnt pathway (enrichment $P=1.23 \times 10^{-3}$; Supplementary Table 14), which activates NFAT via calcineurin signaling ${ }^{42,43}$. Interestingly, LRH-1, the gene product of NR5A2 (Supplementary Box 1), has been shown to bind to $\beta$-catenin ${ }^{44}$, which is also part of the Wnt pathway. Recent findings also suggest that SCZ and BD are characterized by abnormal Wnt gene expression and plasma protein levels, suggesting that suggest that drugs targeting the Wnt signaling pathway may play a role in the treatment of severe mental disorders ${ }^{45}$. Thus, the Wnt signaling pathway and NFAT activation are the most likely candidate pathways for IBD and SCZ, where risk is mediated by gene expression.

\section{Discussion}


By performing cross-tissue and multiple-gene conditioned transcriptome-wide association studies (TWAS) for 23 tissues of the GBA for three clinically related inflammatory diseases of the gastrointestinal tract (CD, UC and PSC) and four diseases of the mind and brain (SCZ, MDD, BD and ADHD) we identified susceptibility genes NR5A2, SATB2, and PPP3CA shared between IBD (CD/UC) and SCZ that are (genetically) expressed both in intestinal and brain tissues. We estimated the proportion of the genetically regulated component of expression $\left(h_{\text {med-min }}{ }^{2}, h_{\text {med-max }}{ }^{2}\right)$ with respect to total gene expression, measured across a maximum of 23 different tissues, to be $(13.4 \%, 18.2 \%)$ for NR5A2, $(6.9 \%, 24.8 \%)$ for SATB2, and $(5.0 \%, 10.9 \%)$ for PPP3CA. These three genes could represent a molecular link between psychiatric and inflammatory traits and may contribute to the observed genetic correlations ${ }^{27,46}$ and increased prevalence of SCZ in CD/UC patients at the population level ${ }^{7-9}$.

Our bioinformatic cross-tissue TWAS pipeline for selected tissues of GBA addressed typical technical drawbacks of previous TWAS studies and enabled conditional analysis of multiple disease-associations at TWAS loci. In our opinion, TWAS analyses for CD, UC and SCZ had the highest statistical power to detect shared TWAS susceptibility genes, as specifically for CD, UC and SCZ a much higher expression-mediated heritability $\left(h_{\text {med }}{ }^{2}\right)$ has recently been estimated than for other psychiatric disorders such as $\mathrm{BD}^{47}$. Another key outcome of our GWAS/TWAS conditional analysis for these three genes is that the expression-disease associations largely explain the previously fine-mapped GWAS association signals at nearby GWAS susceptibility loci for SCZ ${ }^{30}$, CD and $\mathrm{UC}^{31,32}$, suggesting that risk is mediated by genetically regulated expression at those loci. Together with the results of our gene set enrichment pathway analysis, we were able to show that genetic variation mediated by gene expression in the Wnt signaling pathway and NFAT activation by calcineurin is associated with both SCZ and IBD.

A very interesting observation is the gene-disease association with PPP3CA. PPP3CA encodes a subunit of calcineurin (the catalytic subunit Calcineurin A). Calcineurin is a $\mathrm{Ca}^{2+/}$ calmodulin-regulated serine/threonine protein phosphatase ${ }^{48}$, which activates the transcription factor NFAT (nuclear factor of activated $\mathrm{T}$ cells) and thus plays a key role in the regulation of the immune response (Fig. 5). After multiple-gene-conditioned fine-mapping analyses at loci with multiple gene-disease association signals, in total, 18 out of 55 genes of the NFAT signaling pathway showed a gene-disease association with either IBD or SCZ, or both (Fig. 5; Methods). Calcineurin inhibitors (e.g. cyclosporine A and tacrolimus) have long been used as immunosuppressants after solid organ transplantation and are used as first-line treatment in PSC patients who have undergone liver transplantation ${ }^{49}$. Calcineurin inhibitors are also being studied for their efficacy in a number of autoimmune diseases ${ }^{50}$. Recent clinical trials with tacrolimus have demonstrated the efficacy and safety of tacrolimus in treatment-resistent $U^{51}$ and ulcerative proctitis (UC confined to the rectum) ${ }^{52,53}$. However, knockout of calcineurin in the forebrain of mice was found to be associated with symptoms of schizophrenia-like psychosis ${ }^{54}$, and recently experiments in rats have shown that calcineurin inhibitor therapy may be a risk factor for the development of neurobehavioral changes ${ }^{55}$. In humans, treatment with calcineurin inhibitors sometimes showed an increase of neuropsychiatric side effects ${ }^{56,57}$ and tracrolimus has been reported to cause relapse of schizophrenia ${ }^{58}$. Calcineurin-inhibiting immunosuppression used after solid organ transplantation have also been associated with multiple neuropsychiatric side effects ${ }^{59}$. Our results therefore suggest that calcineurin inhibitors should be used with caution in psychiatrically predisposed IBD patients and that transplanted PSC-IBD patients using calcineurin inhibitors as first-line immunosuppressive treatment may be at increased risk of psychiatric side effects. Consistent with these results from clinical and functional studies, we found that decreased predicted expression of PPP3CA is associated with increased risk for SCZ (Table 2).

Our analysis of bulk RNA-Seq and scRNA-Seq data showed that PPP3CA expression is mainly restricted to enteroendocrine and Paneth-like cells of the ileum, colon, and rectum. The gut microbiota produces various metabolites (including short chain fatty acids, secondary bile acids and lipopolysaccharides) that modulate enteroendocrine cells and produce hormonal signals reflecting food intake, microbial composition and epithelial integrity ${ }^{60}$. Paneth cells are secretory epithelial cells of the intestine that contain antibacterial proteins and alpha-defensins that are released into the intestinal lumen in response to a series of stimuli ${ }^{61}$, indicating a possible link to the GBA. PPP3CA is also expressed in the brain, there is evidence for a general implication in SCZ, and studies show more calcineurin immunoreactive neurons in the caudate nucleus of SCZ patients (Supplementary Box 1), which, together with the putamen where we found the TWAS gene-disease association, forms the striatum. Taken together with the literature (Supplementary Box 1), PPP3CA has probably at least two distinct functions relevant to SCZ or IBD, one in neuronal signal transduction in the striatum and the other in signal transduction for immune modulation in Paneth and enteroendocrine cells. NR5A2 (encoding human LRH-1) regulates intestinal glucocorticoid synthesis and is known to protect epithelial integrity and attenuate inflammatory damage in murine and human intestinal organoids, including those derived from IBD patients (Supplementary Box 1). Although NR5A2 appears to be nearly absent from the brain and blood based on the present data, there is evidence of its function in brain tissues (Supplementary Box 1), so we suspect that NR5A2 expression is restricted to less-studied regions of the brain and that it has separate functions in the gut and brain. SATB2 is a promising candidate for a GBA susceptibility gene because it is predominantly expressed in the colon sigmoideum and prefrontal cortex, consistent with our TWAS results in which SATB2 expression is associated with SCZ and UC but not with CD. SATB2 is also a prognostic marker in UC-associated colorectal cancer (Supplementary Box 1). In summary, gene expression analyses for NR5A2, SATB2, and PPP3CA using intestinal and brain tissue data from different developmental stages and from scRNA-seq studies support the findings from previous human, mouse, and organoid studies and suggest a pleiotropic role for these genes as part of the GBA.

\section{Methods}

\section{Consortium GWAS summary statistics}

Crohn's disease (CD) and ulcerative colitis (UC) case and control cohorts (Supplementary Table 1) from 15 countries across Europe, North America and Australia have previously been described, quality controlled (QCed) and genotype imputed ${ }^{12,31}$. Primary sclerosing cholangitis (PSC) case and control cohorts from 14 countries in Europe and North America and have previously been described, QCed and imputed ${ }^{12,62}$. Number of overlapping (duplicate) GWAS samples for CD, UC and PSC GWAS data sets were determined by identity-by-descent (IBD) analysis ${ }^{12}$. Because of a partial sample overlap in cases and controls between Immunochip and GWAS data sets for CD, UC and PSC (Supplementary Table 1), the smaller sample-sized GWAS data sets for CD, UC and 
PSC (data sets \#2, \#4, \#6 in Supplementary Table 1) were used only for validation of TWAS association results from the TWAS discovery phase (see text below). Schizophrenia (SCZ), Major depressive disorder (MDD), Bipolar disorder (BD) and Attention deficit/hyperactivity disorder (ADHD) imputed GWAS summary statistics were used as described elsewhere ${ }^{63-66}$ and were downloaded from https://www.med.unc.edu/pgc/download-results/.

\section{Selection of tissues for TWAS of the GBA}

Recently, a heritability enrichment analysis of specifically expressed genes across 205 tissues and cell types in combined with GWAS summary statistics of $C D, U C, S C Z, M D D, B D$ and $A D H D$ and subsequent validation of enrichments results with chromatin data yielded statistically significant enrichment results for tissues of the central nervous system (CNS) for psychiatric diseases and for tissues from blood/immune cell types and the gastrointestinal tract for inflammatory bowel diseases ${ }^{18}$. Based on these results, we restricted our TWAS analyses using genome-wide and transcriptome-wide reference data of 4,043 samples from brain, immune cell and the gastrointestinal tissues (Supplementary Table 2) available from consortia projects GTEx ${ }^{19}$ (version V7, dbGaP accession code phs000424.v7.p2), STARNET ${ }^{20}$ (dbGaP accession code phs001203.v1.p1), and BLUEPRINT ${ }^{21} \mathrm{ftp} / / / \mathrm{ftp} . \mathrm{ebi}^{\mathrm{ac} . u k / p u b / d a t a b a s e s / b l u e p r i n t /) .}$ As described by Hu et al. ${ }^{15}$, biallelic SNPs with minor allele frequency (MAF) $>=0.01$ were retained and normalized gene expression information was adjusted to correct for potentially confounding effects of sex, sequencing platform and the three main principal components from principal component analysis (PCA) of the genome-wide genotype data, and with estimation of expression residuals (PEER) factors ${ }^{67}$.

\section{UTMOST expression imputation models}

To estimate the genetically regulated expression of each gene in the genome across 23 tissues of the GBA, we applied multivariate-response penalized regression models as implemented in UTMOST (https://github.com/Joker-Jerome/UTMOST) to predict cross-tissue gene expression from reference data sets. Briefly, a standard least-squares loss function was minimized, with a L1 Lasso penalty adaptively set based on sample sizes to select predictive SNP variables in a range of $\pm 1 \mathrm{Mb}$ around each gene and force shrinkage in effect size estimation (within-tissue effects), and further with a group-Lasso penalty set across multiple tissues on the effect sizes of each SNP to select eQTLs shared between tissues (cross-tissue effects) ${ }^{15}$. Such cross-tissue models for expression imputation favor eQTLs that are shared across tissues while preserving tissue-specific effects, and UTMOST's approximation procedure proposed for coordinate descent iteration handles incomplete data in the reference expression matrix.

\section{Expression imputation, association testing and GBJ test}

UTMOST was further used to test for gene-based association at the level of gene expression regulation, taking into account cross-tissue regulatory effects. A univariate regression model was applied to test the association between predicted gene expression (i.e., the genetically regulated component of gene expression) and disease status for each tissue separately (although previously trained over several tissues at the same time). To summarize the tissuespecific results and test whether a gene is significant for at least one tissue, a generalized Berk-Jones (GBJ) test ${ }^{22}$ was used to combine associations across individual tissues into a unified test of association. The GBJ is based on the absolute values of gene trait $Z$ score, allowing for directions of eQTL effects in the different tissues, and uses tissue covariance to correctly weight results across tissues and correct for multiple tissue testing. We used the Bonferroni correction on the number of genes tested to determine transcriptome-wide significance $\left(P<0.05 / 15,587=3.20 \times 10^{-6}\right)$. For the CD, UC and PSC phenotypes, it had to hold additionally that transcriptome-wide significant genes replicate with $P<0.05$ in the smaller GWAS datasets \#2, \#4, \#6 in Supplementary Table 1.

\section{Comparison with other pre-trained single-tissue expression imputation models}

The elastic net approach from S-PrediXcan ${ }^{23}$ was used for expression imputation of 19 GTEx tissues (Supplementary Table 2) followed by gene-disease association testing using logistic regression for our 10 study data sets (Supplementary Table 1). The precomputed reference based on GTEx v7 data was available at http://predictdb.org/. In addition, FUSION's ${ }^{24}$ Bayesian linear mixed model was used for expression imputation and gene-disease association testing with precomputed weights derived from same 19 GTEx tissues v7 (http://gusevlab.org/projects/fusion/). The number of Single-tissue marginal significant genes $\left(P<3.20 \times 10^{-6}\right)$ from UTMOST, FUSION and S-PrediXcan for the 19 GTEx tissues and the 10 GWAS studies included in this study were compared with the number of reference samples used for gene expression imputation training. The number of reference samples used in expression imputation training was taken from the supplementary information or the databases of the respective publications; it is possible that the number of GTEx v7 reference samples differs for the different tools due to different pre-filtering analyses. For all tools, only the marginal, uncorrected, p-values were considered for benchmark purposes. Spearman correlation and a simple linear regression model were computed separately for each of the 10 studies. A paired MannWhitney U-test was applied to test for differences in slopes $(\beta)$ between the three tools.

\section{Cross-tissue multiple-locus-genes conditional analysis and GBJ test}

We used the UTMOST multiple regression approach to perform multiple-locus-genes-conditional cross-tissue analysis for each transcriptome-wide significant gene (from unconditioned analysis) in each of the 10 studies separately to prioritize gene-disease associations at the same locus within the TWAS locus boundaries of the $\pm 1 \mathrm{Mb}$ range. Conditional analysis accounts for one of the main problems of the TWAS gene-based association test, namely the potential coregulation of multiple genes by the same eQTLs or the presence of independent eQTLs across genes that are in linkage disequilibrium (LD) ${ }^{14}$. Again, the association statistics of the conditional analysis were combined across tissues using the generalized Berk-Jones score (GBJ) test described above. Again, we used the Bonferroni correction on the number of genes tested to determine transcriptome-wide significance $\left(P<0.05 / 15,587=3.20 \times 10^{-6}\right)$.

\section{Overlap of gene-disease associations with the boundaries of known susceptibility loci from genome-wide association studies (GWAS) for CD, UC, PSC, SCZ, MDD, BD, ADHD}


For genomic position comparison, locus boundaries of established GWAS loci were adopted from the last fine-mapping GWAS studies for SCZ ${ }^{30}$ and $\mathrm{CD} / \mathrm{UC}^{31,32}$. A gene was labeled "Overlap with GWAS locus" in Table 2 if the gene had an overlap with an established GWAS locus. Gene coordinates (GRCh37, Jan 2020) were obtained using the Ensembl database and BioMart ${ }^{68}$.

\section{Comorbidity analysis in the Danish National Patient Registry (DNPR)}

To determine significant co-occurrences (disease-pairs) for the seven diseases under study, we screened an independent data set covering ICD10 diagnosis codes from 7,191,385 people of the entire Danish population in the period from 1994 to $2018^{69}$. The DNPR includes primary and secondary diagnoses coded according to the International Statistical Classification of Diseases 10th Revision (ICD-10). We identified patients diagnosed with one of the seven disease and then a control group of 20 controls for each case, matched by sex and year of birth. The strength of the association between the diseases was assessed by relative risk (RR). P-values were calculated using a two-sided Fisher exact test and corrected for multiple testing using the false discovery rate (FDR).

\section{Trait correlation at the level of predicted gene expressions}

Genes are often co-regulated, i.e. they either share the same eQTLs or eQTLs exist in strong LD. For this reason, we used a subset of 1,825 co-regulated and eQTL-independent genes recently provided for Mendelian randomization studies ${ }^{26}$, and further excluded genes from the extended MHC region (chr6:25-34Mb) to avoid inflated correlation measures. Spearman's correlation of all traits with each other was calculated separately for each of the 23 tissues based on the single-tissue marginal Z scores (Supplementary Table 9) and additionally based on the GBJ-test marginal test-statistics (Supplementary Table 9; Fig. 2).

\section{Mendelian randomization (MR) analysis at the level of predicted gene expressions}

We performed multi-gene MR analysis at the level of predicted gene expressions using GSMR ${ }^{29}$ (Generalised Summary-data-based Mendelian Randomisation) to test for a causal association between of one trait (exposure) on another (outcome) using summary-level TWAS gene association statistics. We used only standardized transcriptome-wide significant marginal UTMOST gene-disease associations as instruments ( $\left.\mathrm{p}_{\text {marginal\&conditional }}<3.2 \mathrm{e}-6\right)$ that were likely independent of neighboring genes by enforcing a minimum distance of $1 \mathrm{Mb}$ between the instrumental genes (see section Cross-tissue multiple-locus-genes conditional analysis). (Results of the conditional GBJ test could not be used because this test does not output a directional effect size ( $Z$ score); thus, MR analysis was performed on our tissue-specific TWAS results.) At least 10 significant, independent gene-disease associations were necessary as instruments to obtain reliable regression results ${ }^{29}$. Because genetically regulated expression is (by definition) heritable and inferred from SNP-disease association effects, the MR approach from GSMR was performed with (statistically independent) TWAS genes. This satisfies the requirements of MR analysis according to Bowden et al. ${ }^{70}$. We further used the HEIDI ((heterogeneity in dependent instrument)-outlier test) ${ }^{29}$ to detect and eliminate pleiotropic gene-disease associations (genetic instruments) that have apparent pleiotropic effects (horizontal pleiotropy, $P_{\mathrm{HEIDI}}<0.01$ ) on both traits (exposure and outcome) under investigation.

\section{Gene overlap analysis}

To find potential genes mediating the gut brain axis in the same or different tissues, we considered as candidates all genes that were transcriptome-wide significant for both one inflammatory and one psychiatric trait (i) in the same tissue or (ii) in the GBJ test, both marginally $\left(P_{\text {marginal }}<3.20 \times 10^{-6}\right)$ and conditionally $\left(P_{\text {conditional }}<3.20 \times 10^{-6}\right)$ significant. Option (ii) is more liberal and thus includes genes, which are significant in one tissue in a psychiatric trait but significant in an inflammatory trait in another tissue.

\section{Gene expression heritability estimation}

Because the heritability of gene expression of each gene was not given by UTMOST expression reference and training files, we extracted heritability estimates of all available tissues from our FUSION results (see section Comparison with other pre-trained single-tissue expression imputation models) by determining the lowest and the highest heritability value per gene, respectively, to give a kind of confidence range of the expected heritability. Briefly, for heritability estimation, the cis locus was defined as $+/-500 \mathrm{~kb}$ of gene boundaries. Samples were restricted to Europeans by principal components analysis (PCA). FUSION's heritability models were adjusted for 20 gene expression principal components from PCA, two genetic ancestry PCs, and local structural variation estimated from SNP array data of reference data sets ${ }^{24}$. Heritability estimation was performed using GCTA/GREML ${ }^{71}$.

\section{GWAS/TWAS conditional analysis}

If a significant TWAS disease gene is present for a given locus, we expect that the eQTLs of this gene explains a portion of the original GWAS signal seen in the input GWAS summary statistics, for example, in the presence of statistically causal protein-coding variants. To estimate the proportion of the GWAS signal explained by the eQTLs of a significant TWAS candidate gene, GWAS summary statistics were conditioned with GCTA COJO ${ }^{72}$, version 1.92 .2 ) using a maximal set of noncolinear eQTL variants of a TWAS gene (of $\pm 1 \mathrm{Mb}$ flanking region around the gene) for each candidate gene of interest. The aim of this analysis was to qualitatively test whether the eQTLs of a transcriptome-wide significant gene were a possible statistical explanation for the GWAS association signal. GCTA COJO uses the allele frequency, beta, standard error of the beta, the association p-value and the population size of the GWAS summary statistics as input. All eQTLs with a non-zero effect of a significant TWAS candidate gene were given to COJO using parameter-select, whose algorithm is designed to find non-colinear associated SNPs (MAF > 0.01) to subsequently condition on. The P value threshold for including disease-associated non-colinear eQTL sets was chosen 0.05 ; this relatively weak association significance threshold was chosen to additionally identify eQTLs from non-genome-wide significant regions. The resulting set of non-colinear eQTL SNPs was used to condition the GWAS summary data using the GCTA COJO parameter -cond via conditional linear regression analysis ${ }^{33}$. The resulting TWAS-conditioned GWAS summary statistic represents the minimal portion of the GWAS signal that is not covered by the eQTLs of the selected gene and thus could not have been included in the TWAS association. This may suggest that the TWAS gene, although significantly associated, may not be sufficient to fully explain the GWAS association at this locus. 
In summary, our complete UTMOST- and GCTA COJO-based analysis can answer three questions on the association of genetically regulated expression of a gene in a given tissue; (i) whether the eQTLs weighted from the UTMOST-trained models provide an explanation for a GWAS signal with \pm 1 Mb around a TWAS candidate gene (marginal UTMOST test), (ii) which TWAS gene is most likely to represent a GWAS signal given multiple associated TWAS genes (conditional UTMOST test), and (iii), whether there are SNP-disease associations in the GWAS data that cannot be explained by eQTLs in LD (COJO test), such that there may be a different eQTL gene, causal (protein-coding) variants, or epigenetic modifications at the locus that result in a GWAS association signal.

\section{Bulk RNA-Seq data of different developmental stages and scRNA-seq expression data lookup for tissues of the GBA}

Median TPM- (transcripts per million) normalized bulk tissue gene expression data from 49 tissues of 838 postmortem donors were retrieved from the data portal of GTEx project ${ }^{73}$ (https://www.gtexportal.org/home/datasets) including (i) a dataset of a total of 297 mRNA libraries from 23 developmental stages from human fetal (elective abortion) to unrelated deceased postnatal, juvenile and adult tissue samples ${ }^{37}$ (E-MTAB-6814,

https://www.ebi.ac.uk/gxa/experiments/E-MTAB-6814/Downloads), (ii) a dataset of 50 mRNA libraries from expression of the human fetal (elective abortion) and juvenile gut biopsies, two different tissues each, from intestinal epithelial organoids and purified intestinal epithelial cells ${ }^{38}$ (E-MTAB-5015,

https://www.ebi.ac.uk/gxa/experiments/E-MTAB-5015/Downloads) and (iii) the BLUEPRINT blood cell reference data from 77 mRNA libraries of 27 cell types from infant to adult donors (E-MTAB-3827, https://www.ebi.ac.uk/gxa/experiments/E-MTAB-3827/Downloads). The obtained gene expression values were gene-wise centered and $Z$ score normalized for visualization in $\mathrm{R}$.

Single cell RNA-seq (scRNA-seq) data of healthy human small intestine, colon and rectum biopsies, from two donors each, with a total of 14,537 cells were retrieved from GSE125970 40 and single cell libraries from five male postmortem donors (3 prefontal cortex samples, 4 hippocampus) from the GTEx biobank (Habib et al; 28846088) were retrieved via https://www.covid19cellatlas.org. Data were processed and log-normalized as described by Sungnak et al. ${ }^{74}$ and visualized using the scanpy v1.6.0 package ${ }^{75}$ (https://scanpy.readthedocs.io/en/stable/api/scanpy.pl.dotplot.html).

\section{NFAT pathway visualization}

The list of 55 genes of the term "Calcineurin-dependent NFAT signaling role in lymphocytes" from the database BioPlanet $2019^{35}$ was downloaded from https://maayanlab.cloud/Enrichr/\#stats ${ }^{76}$ (May 25st 2021) and used as input for GeneMania ${ }^{77}$ with no genes added and all available physical interaction edges, pathway-based edges and colocalization edges plotted. The resulting network was exported and imported into Cytoscape $3.8 .2^{78}$ to adjust the visual style. We chose both marginal and conditional nominal significance $\left(P_{\mathrm{GBJ}}\right.$ conditional $\left.<0.05\right)$ as the threshold for highlighting genes.

\section{Declarations}

\section{Competing interests}

The authors have no competing interests or other interests that might be perceived to influence the results and/or discussion reported

\section{Author contributions}

D.E. was responsible for the concept and the design of the study. T.F., A.F., M.M.N., F.D., T.H.K., S.S., C.S., P.H., P.K. assisted with recruitment or phenotyping of patients in the inflammatory and psychiatric disease clinical cohorts from which the GWAS summary statistics were derived or provided GWAS summary statistics. F.U-W. and D.E. implemented statistical models and performed computation analyses. C.M., O.B., E.M.W., I.F.J., S.Be., O.W., K.B. and S.Br. helped with bioinformatic analyses. S.J. and F.U-W. performed RNA-sequencing data analysis. T.A.S. designed Fig. 1. D.E. and F.U-W. curated and interpreted results and wrote the manuscript. All authors reviewed, edited and approved the final manuscript.

\section{Acknowledgements}

This work was supported by the German Federal Ministry of Education and Research (BMBF) within the framework of the e:Med Vernetzungsfonds funding concept (GB-XMAP grants 01ZX1709A, 01ZX1709B, 01ZX1709C). The study received infrastructure support from the Deutsche Forschungsgemeinschaft (DFG, German Research Foundation) Cluster of Excellence 2167 "Precision Medicine in Chronic Inflammation (PMI)" (EXC 2167-390884018). Access to the DNPR was approved by the Danish Health Data Authority (FSEID-00003092 and FSEID-00004491 and supported by Novo Nordisk Foundation (grants NNF14CC0001 and NNF170C0027594). We thank the PGC for making the GWAS summary statistics publicly available. We thank the IIBDGC and IPSCSG for permission to use the GWAS/Immunochip summary statistics; a complete list of members and affiliations of the IIBDGC and the IPSCSG can be found in the Supplementary Information. Figure 5b was generated using BioRender.com and a template from Akiko Iwasaki, PhD and Ruslan Medzhitov, PhD.

\section{References}

1. Heiss, C.N. \& Olofsson, L.E. The role of the gut microbiota in development, function and disorders of the central nervous system and the enteric nervous system. J Neuroendocrino/31, e12684 (2019).

2. Skonieczna-Zydecka, K., Marlicz, W., Misera, A., Koulaouzidis, A. \& Loniewski, I. Microbiome-The Missing Link in the Gut-Brain Axis: Focus on Its Role in Gastrointestinal and Mental Health. J Clin Med 7(2018). 
3. Kan, J.M., Cowan, C.S.M., Ooi, C.Y. \& Kasparian, N.A. What can the gut microbiome teach us about the connections between child physical and mental health? A systematic review. Dev Psychobiol 61, 700-713 (2019).

4. Ming, X., et al. A Gut Feeling: A Hypothesis of the Role of the Microbiome in Attention-Deficit/Hyperactivity Disorders. Child Neurol Open 5, 2329048X18786799 (2018).

5. Severance, E.G., Yolken, R.H. \& Eaton, W.W. Autoimmune diseases, gastrointestinal disorders and the microbiome in schizophrenia: more than a gut feeling. Schizophr Res 176, 23-35 (2016).

6. Hartono, J.L., Mahadeva, S. \& Goh, K.L. Anxiety and depression in various functional gastrointestinal disorders: do differences exist? J Dig Dis 13, 252257 (2012).

7. Marrie, R.A., et al. Increased incidence of psychiatric disorders in immune-mediated inflammatory disease. J Psychosom Res 101, 17-23 (2017).

8. Bernstein, C.N., et al. Increased Burden of Psychiatric Disorders in Inflammatory Bowel Disease. Inflamm Bowel Dis 25, 360-368 (2019).

9. Thavamani, A., Umapathi, K.K., Khatana, J. \& Gulati, R. Burden of Psychiatric Disorders among Pediatric and Young Adults with Inflammatory Bowel Disease: A Population-Based Analysis. Pediatr Gastroenterol Hepatol Nutr 22, 527-535 (2019).

10. Bonaz, B.L. \& Bernstein, C.N. Brain-gut interactions in inflammatory bowel disease. Gastroenterology 144, 36-49 (2013).

11. Karlsen, T.H. \& Boberg, K.M. Update on primary sclerosing cholangitis. Journal of hepatology 59, 571-582 (2013).

12. Ellinghaus, D., et al. Analysis of five chronic inflammatory diseases identifies 27 new associations and highlights disease-specific patterns at shared loci. Nat Genet 48, 510-518 (2016).

13. Cross-Disorder Group of the Psychiatric Genomics, C., et al. Genetic relationship between five psychiatric disorders estimated from genome-wide SNPs. Nat Genet 45, 984-994 (2013).

14. Wainberg, M., et al. Opportunities and challenges for transcriptome-wide association studies. Nat Genet 51, 592-599 (2019).

15. Hu, Y., et al. A statistical framework for cross-tissue transcriptome-wide association analysis. Nat Genet 51, 568-576 (2019).

16. Fryett, J.J., Morris, A.P. \& Cordell, H.J. Investigation of prediction accuracy and the impact of sample size, ancestry, and tissue in transcriptome-wide association studies. Genetic epidemiology 44, 425-441 (2020).

17. Gamazon, E.R., Zwinderman, A.H., Cox, N.J., Denys, D. \& Derks, E.M. Multi-tissue transcriptome analyses identify genetic mechanisms underlying neuropsychiatric traits. Nat Genet 51, 933-940 (2019).

18. Finucane, H.K., et al. Heritability enrichment of specifically expressed genes identifies disease-relevant tissues and cell types. Nat Genet 50, 621-629 (2018)

19. Consortium, G.T., et al. Genetic effects on gene expression across human tissues. Nature 550, 204-213 (2017).

20. Franzen, O., et al. Cardiometabolic risk loci share downstream cis- and trans-gene regulation across tissues and diseases. Science 353, 827-830 (2016).

21. Chen, L., et al. Genetic Drivers of Epigenetic and Transcriptional Variation in Human Immune Cells. Cell 167, 1398-1414 e1324 (2016).

22. Sun, R., Hui, S., Bader, G.D., Lin, X. \& Kraft, P. Powerful gene set analysis in GWAS with the Generalized Berk-Jones statistic. PLoS genetics 15 , e1007530 (2019).

23. Barbeira, A.N., et al. Exploring the phenotypic consequences of tissue specific gene expression variation inferred from GWAS summary statistics. Nature communications 9,1825 (2018).

24. Gusev, A., et al. Integrative approaches for large-scale transcriptome-wide association studies. Nat Genet 48, 245-252 (2016).

25. Hirschhorn, J.N. \& Daly, M.J. Genome-wide association studies for common diseases and complex traits. Nature reviews. Genetics 6, 95-108 (2005).

26. Porcu, E., et al. Mendelian randomization integrating GWAS and eQTL data reveals genetic determinants of complex and clinical traits. Nature communications 10, 3300 (2019).

27. Tylee, D.S., et al. Genetic correlations among psychiatric and immune-related phenotypes based on genome-wide association data. Am J Med Genet $B$ Neuropsychiatr Genet 177, 641-657 (2018).

28. Davey Smith, G. \& Hemani, G. Mendelian randomization: genetic anchors for causal inference in epidemiological studies. Hum Mol Genet 23, R89-98 (2014).

29. Zhu, Z., et al. Causal associations between risk factors and common diseases inferred from GWAS summary data. Nature communications 9 , 224 (2018).

30. Pardinas, A.F., et al. Common schizophrenia alleles are enriched in mutation-intolerant genes and in regions under strong background selection. Nat Genet 50, 381-389 (2018).

31. Liu, J.Z., et al. Association analyses identify 38 susceptibility loci for inflammatory bowel disease and highlight shared genetic risk across populations. Nat Genet 47, 979-986 (2015).

32. Huang, H., et al. Fine-mapping inflammatory bowel disease loci to single-variant resolution. Nature 547, 173-178 (2017).

33. Yang, J., et al. Conditional and joint multiple-SNP analysis of GWAS summary statistics identifies additional variants influencing complex traits. Nat Genet 44, 369-375, S361-363 (2012).

34. Bayrer, J.R., et al. LRH-1 mitigates intestinal inflammatory disease by maintaining epithelial homeostasis and cell survival. Nature communications 9 , 4055 (2018)

35. Liu, J., et al. Calcineurin is a common target of cyclophilin-cyclosporin A and FKBP-FK506 complexes. Cell 66, 807-815 (1991).

36. Rehn, A.E. \& Rees, S.M. Investigating the neurodevelopmental hypothesis of schizophrenia. Clin Exp Pharmacol Physio/32, 687-696 (2005).

37. Cardoso-Moreira, M., et al. Gene expression across mammalian organ development. Nature 571, 505-509 (2019).

Page $12 / 18$ 
38. Kraiczy, J., et al. DNA methylation defines regional identity of human intestinal epithelial organoids and undergoes dynamic changes during development. Gut 68, 49-61 (2019).

39. Habib, N., et al. Massively parallel single-nucleus RNA-seq with DroNc-sEq. Nature methods 14, 955-958 (2017).

40. Wang, Y., et al. Single-cell transcriptome analysis reveals differential nutrient absorption functions in human intestine. J Exp Med 217(2020).

41. Kuleshov, M.V., et al. Enrichr: a comprehensive gene set enrichment analysis web server 2016 update. Nucleic Acids Res 44, W90-97 (2016).

42. Huang, R., et al. The NCATS BioPlanet - An Integrated Platform for Exploring the Universe of Cellular Signaling Pathways for Toxicology, Systems Biology, and Chemical Genomics. Front Pharmacol 10, 445 (2019).

43. Rieger, M.E., et al. p300/beta-Catenin Interactions Regulate Adult Progenitor Cell Differentiation Downstream of WNT5a/Protein Kinase C (PKC). J Biol Chem 291, 6569-6582 (2016).

44. Yumoto, F., et al. Structural basis of coactivation of liver receptor homolog-1 by beta-catenin. Proceedings of the National Academy of Sciences of the United States of America 109, 143-148 (2012).

45. Hoseth, E.Z., et al. Exploring the Wnt signaling pathway in schizophrenia and bipolar disorder. Transl Psychiatry 8, 55 (2018).

46. Pickrell, J.K., et al. Detection and interpretation of shared genetic influences on 42 human traits. Nat Genet 48, 709-717 (2016).

47. Yao, D.W., O'Connor, L.J., Price, A.L. \& Gusev, A. Quantifying genetic effects on disease mediated by assayed gene expression levels. Nat Genet 52, 626$633(2020)$.

48. Hubbard, M.J. \& Klee, C.B. Functional domain structure of calcineurin A: mapping by limited proteolysis. Biochemistry 28, 1868-1874 (1989).

49. Yeh, H. \& Markmann, J.F. Transplantation: Are calcineurin inhibitors safer than mTOR inhibitors? Nat Rev Nephro/ 9, 11-13 (2013).

50. Bendickova, K., Tidu, F. \& Fric, J. Calcineurin-NFAT signalling in myeloid leucocytes: new prospects and pitfalls in immunosuppressive therapy. EMBO Mol Med 9, 990-999 (2017).

51. Matsuoka, K., et al. Tacrolimus for the Treatment of Ulcerative Colitis. Intest Res 13, 219-226 (2015).

52. Smith, R.W., H.; Morgan L.; Parkes M.; Lee J.C. Tacrolimus suppositories: a safe and effective treatment for treatment-refractory proctitis. Journal of Crohn's and Colitis 13, S408-S409 (2019).

53. Jaeger, S.U., et al. Tacrolimus Suppositories in Therapy-Resistant Ulcerative Proctitis. Inflamm Intest Dis 3, 116-124 (2019).

54. Takao, K. \& Miyakawa, T. Investigating gene-to-behavior pathways in psychiatric disorders: the use of a comprehensive behavioral test battery on genetically engineered mice. Ann N Y Acad Sci 1086, 144-159 (2006).

55. Brosda, J., et al. Treatment with the calcineurin inhibitor and immunosuppressant cyclosporine A impairs sensorimotor gating in Dark Agouti rats. Psychopharmacology (Berl) 238, 1047-1057 (2021).

56. Chang, S.H., Lim, C.S., Low, T.S., Chong, H.T. \& Tan, S.Y. Cyclosporine-associated encephalopathy: a case report and literature review. Transplant Proc 33, 3700-3701 (2001).

57. Lang, U.E., et al. Immunosuppression using the mammalian target of rapamycin (mTOR) inhibitor everolimus: pilot study shows significant cognitive and affective improvement. Transplant Proc 41, 4285-4288 (2009).

58. Lin, Y., Sun, I.W., Liu, S.I., Loh el, W. \& Lin, Y.C. Tacrolimus ointment-induced relapse of schizophrenia: a case report. Int J Neuropsychopharmacol 10, 851854 (2007).

59. Heinrich, T.W. \& Marcangelo, M. Psychiatric issues in solid organ transplantation. Harv Rev Psychiatry 17, $398-406$ (2009).

60. Gribble, F.M. \& Reimann, F. Function and mechanisms of enteroendocrine cells and gut hormones in metabolism. Nat Rev Endocrino/15, 226-237 (2019).

61. Keshav, S. Paneth cells: leukocyte-like mediators of innate immunity in the intestine. J Leukoc Bio/ 80, 500-508 (2006).

62. Ji, S.G., et al. Genome-wide association study of primary sclerosing cholangitis identifies new risk loci and quantifies the genetic relationship with inflammatory bowel disease. Nat Genet 49, 269-273 (2017).

63. Schizophrenia Working Group of the Psychiatric Genomics, C. Biological insights from 108 schizophrenia-associated genetic loci. Nature 511, 421-427 (2014).

64. Wray, N.R., et al. Genome-wide association analyses identify 44 risk variants and refine the genetic architecture of major depression. Nat Genet $50,668-$ 681 (2018).

65. Stahl, E.A., et al. Genome-wide association study identifies 30 loci associated with bipolar disorder. Nat Genet 51, 793-803 (2019).

66. Demontis, D., et al. Discovery of the first genome-wide significant risk loci for attention deficit/hyperactivity disorder. Nat Genet 51, 63-75 (2019).

67. Stegle, O., Parts, L., Durbin, R. \& Winn, J. A Bayesian framework to account for complex non-genetic factors in gene expression levels greatly increases power in eQTL studies. PLoS Comput Bio/ 6, e1000770 (2010).

68. Smedley, D., et al. The BioMart community portal: an innovative alternative to large, centralized data repositories. Nucleic Acids Res 43, W589-598 (2015).

69. Siggaard, T., et al. Disease trajectory browser for exploring temporal, population-wide disease progression patterns in 7.2 million Danish patients. Nature communications 11, 4952 (2020).

70. Bowden, J., Davey Smith, G. \& Burgess, S. Mendelian randomization with invalid instruments: effect estimation and bias detection through Egger regression. Int J Epidemiol 44, 512-525 (2015).

71. Yang, J., et al. Common SNPs explain a large proportion of the heritability for human height. Nat Genet 42, 565-569 (2010).

72. Yang, J., Lee, S.H., Goddard, M.E. \& Visscher, P.M. GCTA: a tool for genome-wide complex trait analysis. Am J Hum Genet 88, 76-82 (2011).

73. Consortium, G.T. The GTEx Consortium atlas of genetic regulatory effects across human tissues. Science 369, 1318-1330 (2020).

Page $13 / 18$ 
74. Sungnak, W., et al. SARS-CoV-2 entry factors are highly expressed in nasal epithelial cells together with innate immune genes. Nat Med 26, 681-687 (2020)

75. Wolf, F.A., Angerer, P. \& Theis, F.J. SCANPY: large-scale single-cell gene expression data analysis. Genome biology 19, 15 (2018).

76. Chen, E.Y., et al. Enrichr: interactive and collaborative HTML5 gene list enrichment analysis tool. BMC bioinformatics 14, 128 (2013).

77. Warde-Farley, D., et al. The GeneMANIA prediction server: biological network integration for gene prioritization and predicting gene function. Nucleic Acids Res 38, W214-220 (2010).

78. Shannon, P., et al. Cytoscape: a software environment for integrated models of biomolecular interaction networks. Genome Res 13, 2498-2504 (2003).

\section{Figures}

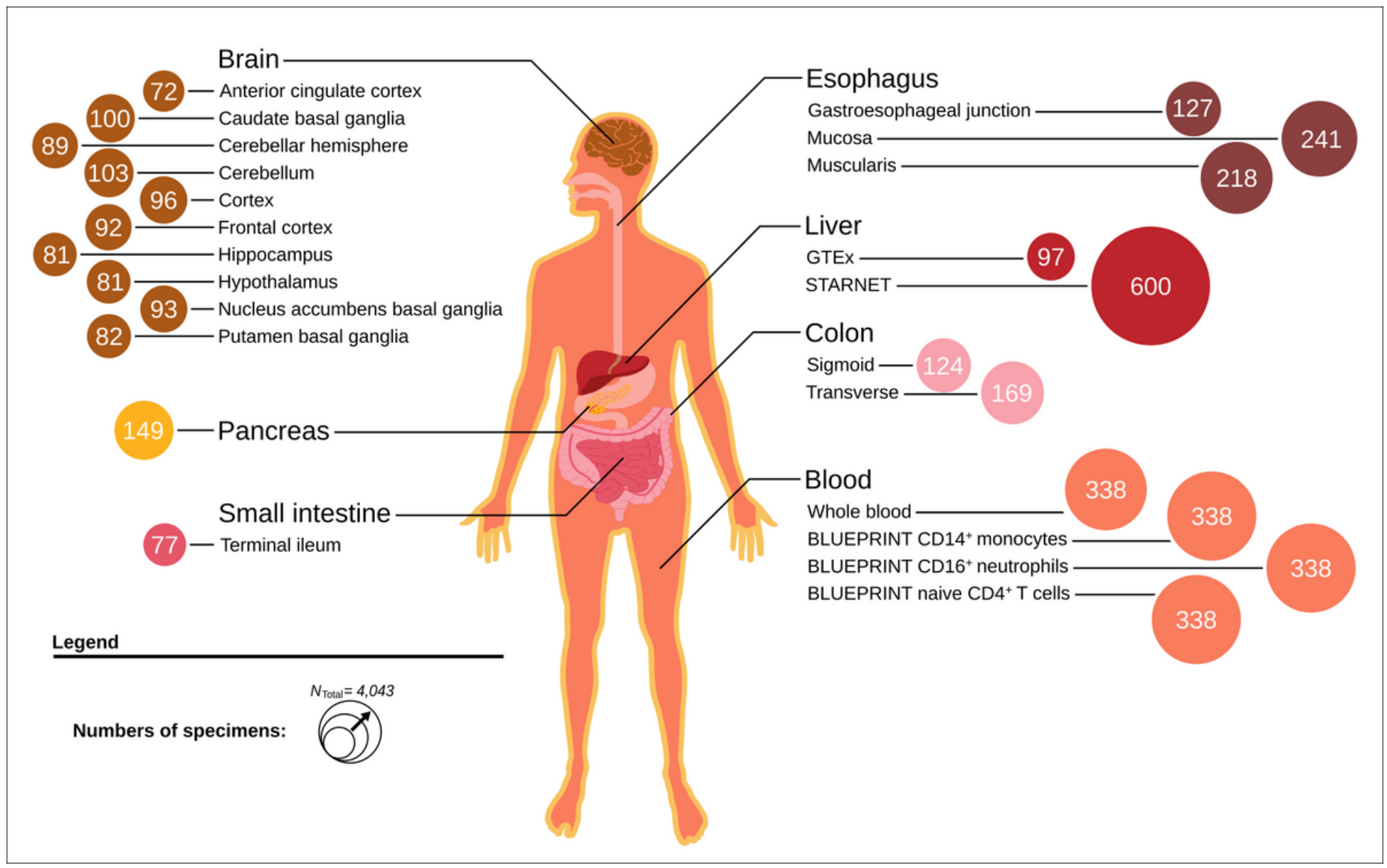

Figure 1

Selection of 23 tissue and cell types to perform transcriptome-wide association analyses (TWAS) for gut-brain axis (GBA) diseases. We selected 23 tissues and cell types previously found to contribute to the heritability of psychiatric or immune-mediated disease (Methods). Cross-tissue TWAS training and imputation were performed using transcriptome-wide and genome-wide reference data from 4,043 reference samples from 23 tissues and cell types (Supplementary Table 2) provided by consortium projects GTEx, STARNET, and BLUEPRINT (Methods): gastrointestinal and liver tissues ( $\mathrm{n}=9$ ), brain tissues $(n=10)$, whole blood $(n=1)$ and immune cell types (CD14+ monocytes, CD16+ neutrophils, CD4+ T-cells; $n=3)$. Different colors represent biological samples from different tissue classes. The size of the circles corresponds to the number of reference samples of the respective tissue. 

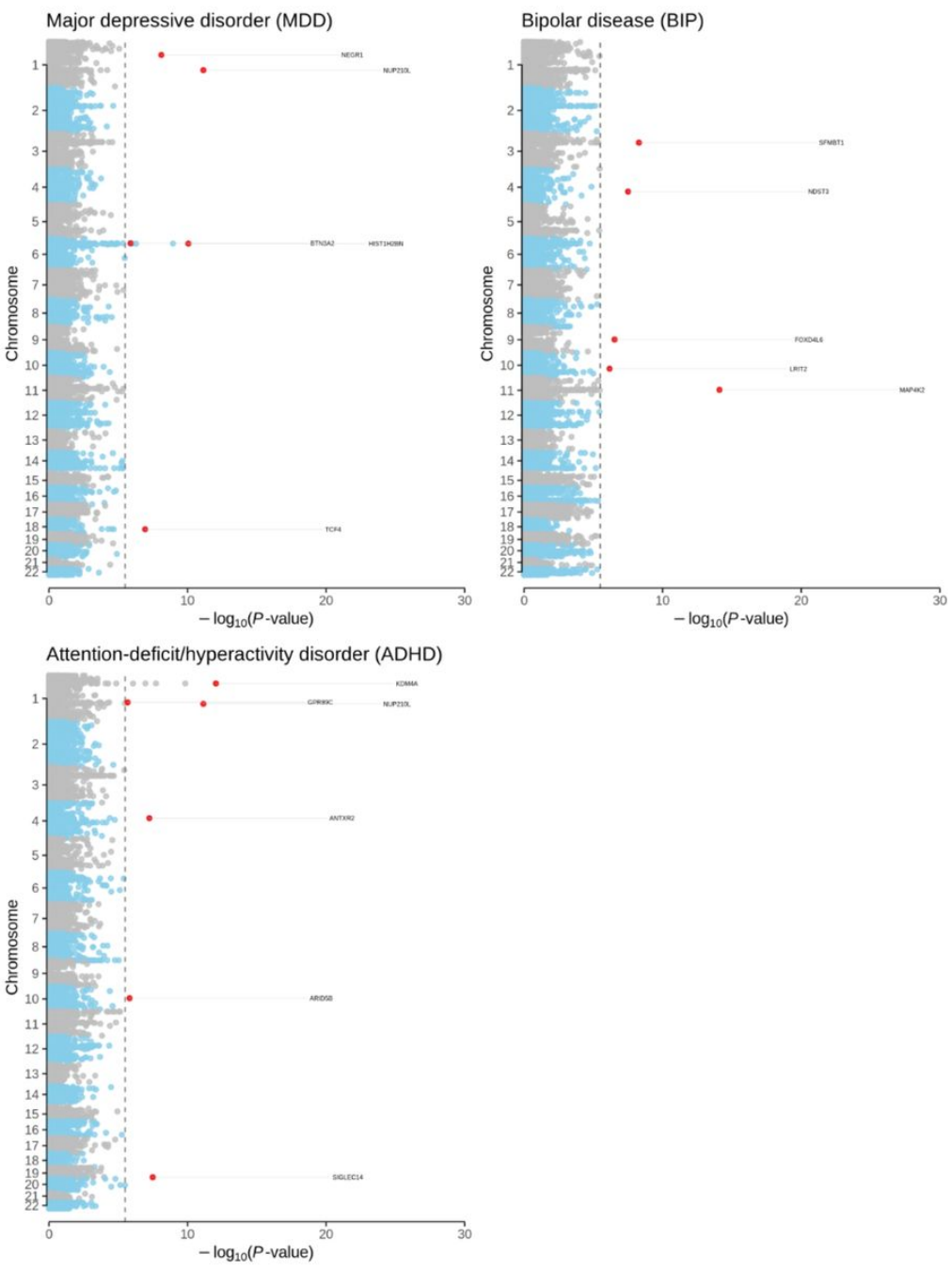

\section{Figure 2}

Manhattan plots showing transcriptome-wide significant (Pconditional<3.20×10-6 from GBJconditional) gene discoveries for psychiatric and immune phenotypes in 23 gut-brain-axis (GBA) tissues, and proportion of shared predicted transcriptome-wide disease-associated gene expression ( $\rho E$ ) for pairs of immune-mediated and psychiatric diseases averaged across 23 tissues of the GBA. a) grey dotted line, transcriptome-wide significance threshold; red dots, lead gene-disease associations at TWAS loci (for regions of size $\pm 1 \mathrm{Mb}$ ) and conditioned on all genes within $\pm 1 \mathrm{Mb}$ regions. CD, Crohn's disease; UC, ulcerative colitis; PSC, primary sclerosing cholangitis; SCZ, schizophrenia; MDD, major depressive disorder; BIP, bipolar disease; ADHD, attention-deficit/hyperactivity disorder; b) Spearman's trait correlation values at the transcriptome-wide level of gene expression for each disease pair and averaged across 23 tissues of the GBA show that effects of genetic risk variants shared between psychiatric and immune phenotypes are largely mediated by gene expression. Strongest positive correlations across psychiatric and immune phenotypes were observed for pairs UC and BP $(\hat{\rho} \mathrm{E}=0.15), \mathrm{CD}$ and $\mathrm{BP}(\hat{\rho} \mathrm{E}=0.14), \mathrm{UC}$ and $\mathrm{SCZ}(\hat{\rho} \mathrm{E}=$ $0.13)$, and $\mathrm{CD}$ and $\mathrm{SCZ}(\hat{\rho} \mathrm{E}=0.11)$, which is in line with genome-wide genetic correlations ( $\hat{\rho} \mathrm{G})$ for pairs $\mathrm{UC}$ and $\mathrm{BP}(\hat{\rho} \mathrm{G}=0.23), \mathrm{CD}$ and $\mathrm{BP}(\hat{\rho} \mathrm{G}=0.22), \mathrm{UC}$ and SCZ ( $\hat{\rho} G=0.14)$, and $C D$ and SCZ ( $\hat{\rho} G=0.12$ ) reported by Tylee et al.27. The dendrogram reflects the corresponding hierarchical single linkage clustering across diseases. Genetic trait correlations for single-tissue results (analysis Single-tissueconditional) are given in Supplementary Table 9 . For selection of 23 tissues, see Figure 1, Supplementary Table 2 and Methods. Results from unconditioned cross-tissue TWAS analysis (GBJmarginal; before multiple-geneconditioned fine-mapping analysis) are shown in Supplementary Figure 2. 

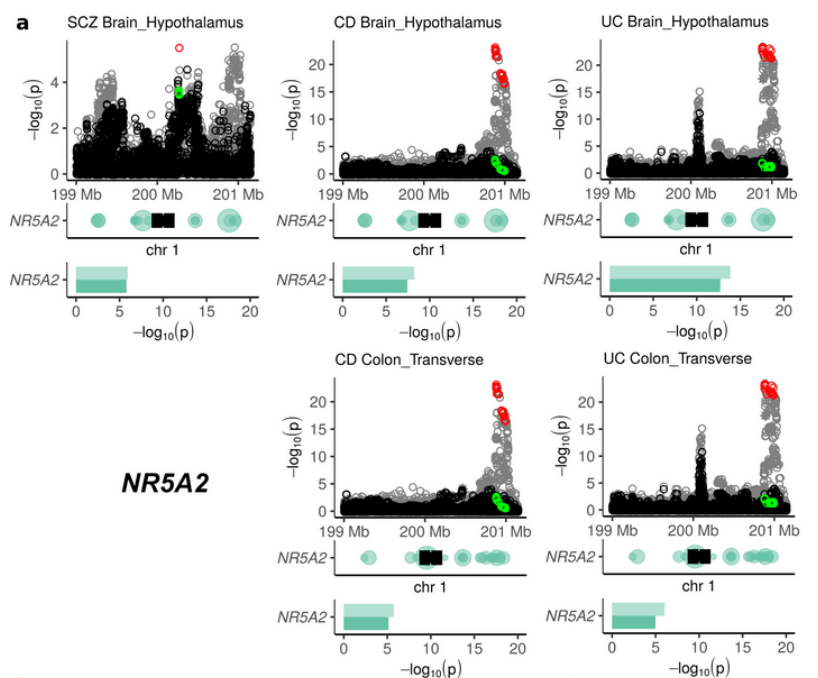

- GWAS SNP statistics

- GWAS fine-mapped SNPS

- GWAS SNP statistics -

- TWAS cond.

- GWAS fine-mapped SNP.

* TWAS gond.

$\mathrm{P}$-value

TWAS $\mathrm{P}$ uncond.

TWAS $\mathrm{P}$ cond.

NR5A2
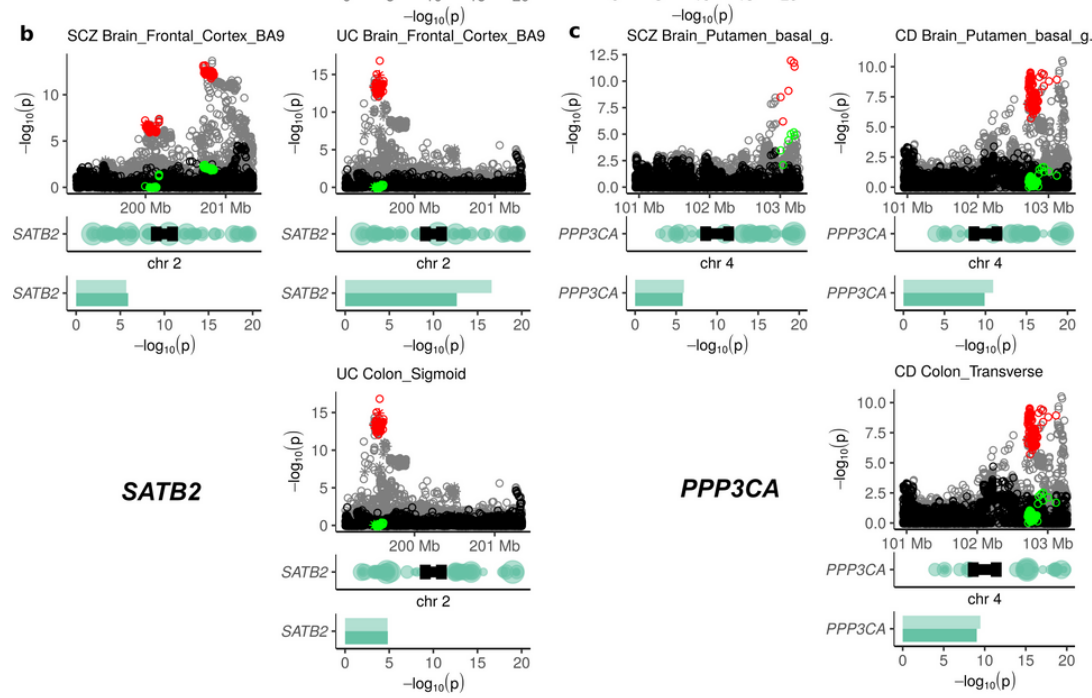

Figure 3

The transcriptome-wide significant gene-disease association signals of NR5A2 (1q32.1), SATB2 (2q33.1) and PPP3CA (4q24) for SCZ, CD and UC largely explain fine-mapped GWAS association signals from nearby GWAS susceptibility loci. (a) The gene-disease association signal of NR5A2 is driven by the nearby but non-overlapping established SCZ and CD/UC GWAS signals located 104 and 654 kilobases (kb) upstream of NR5A2, respectively (Table 2). Increased genetically regulated expression of NR5A2 in hypothalamus and colon transversum was associated with increased risk for schizophrenia (SCZ), Crohn's disease (CD) and ulcerative colitis (UC). (b) The association signal of SATB2 is driven by the nearby but non-overlapping established SCZ and UC GWAS signals located 67 and 424 kilobases $(\mathrm{kb})$ downstream and upstream of SATB2, respectively. Increased genetically regulated expression of SATB2 in frontal cortex BA9 and colon sigmoideum is associated with increased risk for SCZ and UC. Although $95 \%$ credible sets of variants most likely to be causal (red circles; upper part) differ between SCZ and UC, the distribution pattern of TWAS gene eQTLs of SATB2 (grey asterisks [upper part] and green transparent circles [middle part]) leads to the shared gene-disease association for SATB2 in both SCZ and UC. (c) The gene-disease association signal of PPP3CA is driven by the nearby (non-overlapping) established SCZ and CD GWAS signals located 280 and 383 kilobases (kb) upstream of SATB2, respectively. Decreased genetically regulated expression of PPP3CA in putamen basal ganglia and colon transversum is associated with increased risk for SCZ and CD. Figure legend: Subplots include original consortium GWAS SNP summary statistics of size $\pm 1 \mathrm{Mb}$ (grey circles; upper part) around the gene, with the $95 \%$ credible sets of variants most likely to be causal at each locus (red circles; upper part) as defined by GWAS fine-mapping studies30,32. SNP summary stats were conditioned (black circles; upper part) on TWAS gene eQTLs (black asterisks [upper part] correspond to the green transparent circles [middle part], with the relative absolute weight of the eQTLs visualized by the size of green transparent circles) to examine if the original GWAS SNP statistics (grey circles [upper part]; grey asterisks correspond to the green transparent circles [middle part]) can be explained by genetically regulated expression of the gene (green rectangles depict unconditioned/conditioned TWAS P-values from Single-tissueconditional analysis; lower part). GWAS SNP summary statistics of $95 \%$ credible sets of variants after joint GWAS/TWAS fine-mapping analysis are depicted as light green circles (upper part). Gene start and gene end positions are marked by dumbbellshaped black bars (middle part). 

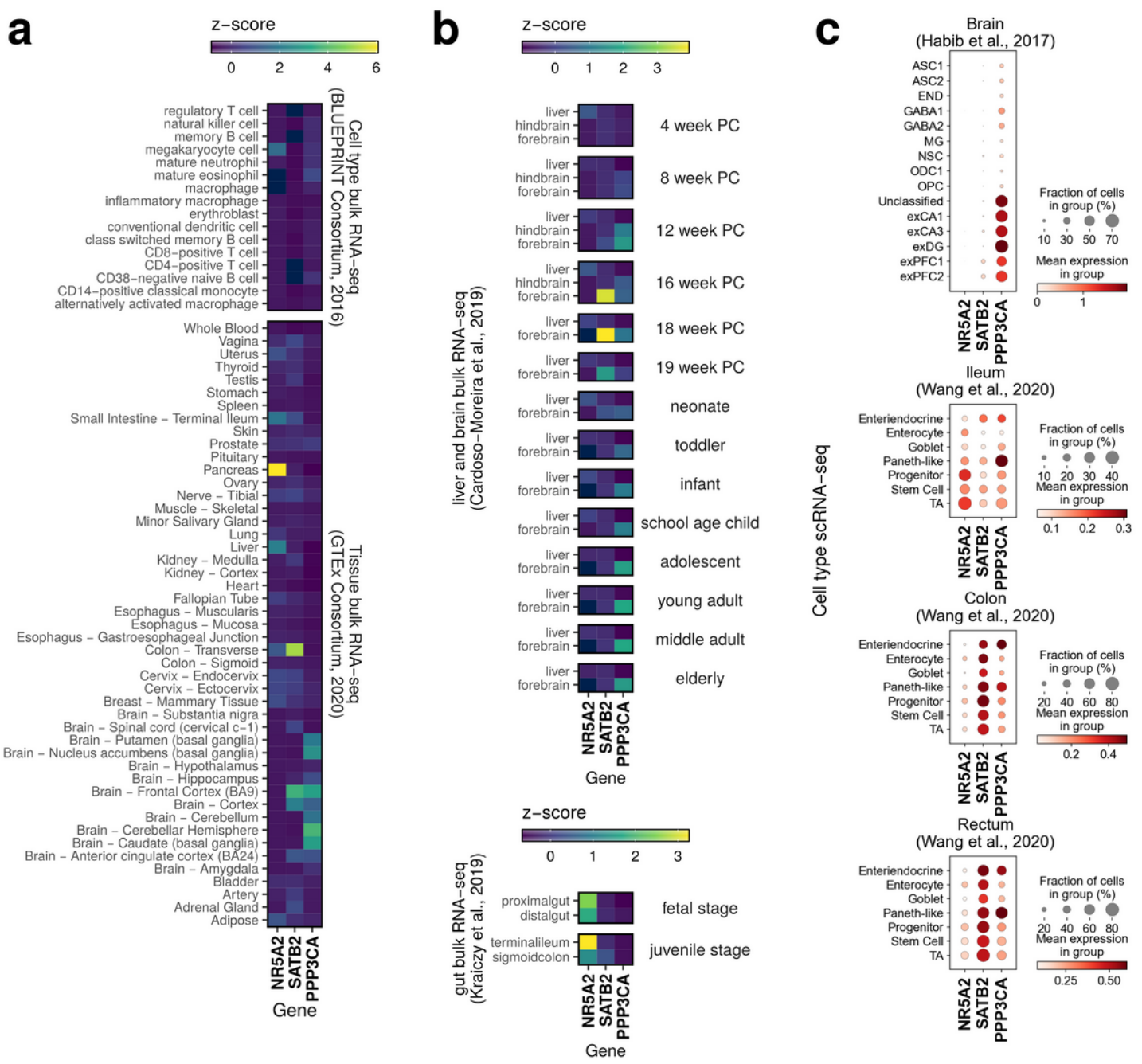

Figure 4

Analysis of bulk and scRNA-seq data suggest a cell-specific pleiotropic role of NR5A2, SATB2, and PPP3CA in SCZ, CD and UC and showed that PPP3CA expression was strongest in neurons and enteroendocrine and Paneth-like cells of the ileum, colon, and rectum, indicating a possible link to the GBA. (A) Screening of several organ- and blood cell type-specific bulk expression data from GTeX and BLUEPRINT shows that NR5A2 is expressed in gastrointestinal tissues but not in the brain, SATB2 is expressed mainly in the colon transversum and prefrontal cortex (the same tissues where we detected the gene-disease associations) and PPP3CA is expressed in several brain tissues but less in intestinal tissues. (B, top) Brain expression data from humans of different ages37 confirm GTeX and BLUEPRINT tissue data and show that NR5A2 is almost absent in brain samples. SATB2 is strongly upregulated in the fetal forebrain in midfetal development. This suggests a developmental function that may predispose to disease when dysregulated. PPP3CA expression is strongest in the forebrain and increases with age. Expression in liver is given for reference. PC, post conception. (B, bottom) Data on expression in the developmental gut38 show that NR5A2 is strongly expressed in the ileum and less so in the colon, whereas SATB2 is more abundant in the colon than in the ileum. This is consistent with the observation that SATB2 is significant for UC but not for CD (Table 2). PPP3CA expression is not evident from bulk RNA-Seq data of intestinal tissue. (C) NR5A2 is expressed in ileal progenitor, stem cells and transient amplifying (TA) cells, less strongly in colon and rectum, and not in brain, consistent with bulk RNA-seq results. SATB2 is strongly expressed in all cell types of the colon and rectum. PPP3CA, despite weak expression in ileum or colon, is enriched in enteroendocrine and Paneth-like cells of the colon and rectum, supporting a role in immunomodulation. In the brain, PPP3CA is enriched in neurons. Overall, these expression data indicate that separate functions are likely in the gut and brain based on specific expression patterns. NR5A2 expression was not found in the brain, but literature results make brain-specific function highly likely (Supplementary Box 1). (exPFC=glutamatergic neurons from the PFC, exCA1/3=pyramidal neurons from the Hip CA region, GABA=GABAergic interneurons, exDG=granule neurons from the Hip dentate gyrus region, $\mathrm{ASC}=$ astrocytes, $\mathrm{NSC}=$ neuronal stem cells, $\mathrm{MG}=$ microglia, $\mathrm{ODC}=$ oligodendrocytes, $\mathrm{OPC}=$ oligodendrocyte precursor cells, $\mathrm{NSC}=$ neuronal stem cells,

$\mathrm{SMC}=$ smooth muscle cells, END= endothelial cells). 


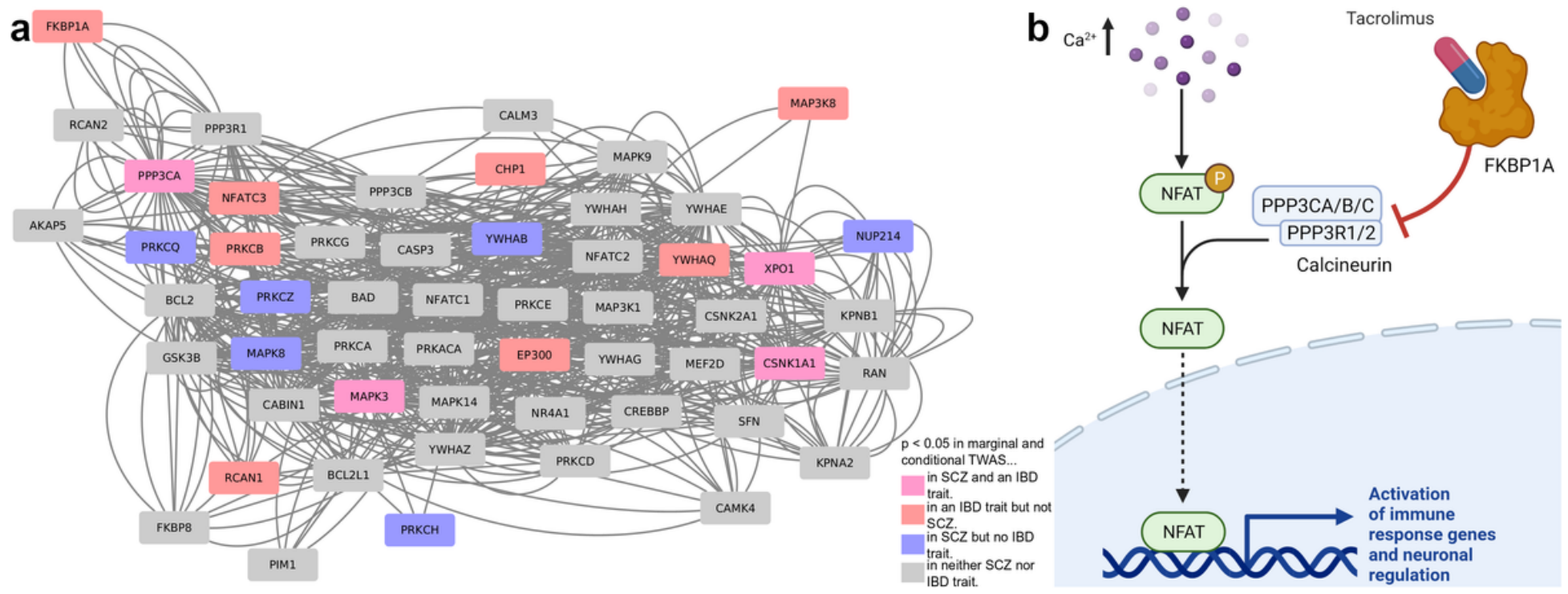

Figure 5

Calcineurin-dependent NFAT signaling as shared signaling pathway for IBD and SCZ. (a) 18 gene-disease associations with suggestive significance (PGBJ conditional<0.05; i.e. after multiple-gene-conditioned fine-mapping analysis) are enriched in the 'Calcineurin-dependent NFAT signaling in leukocytes' pathway (comprising 55 genes; Methods), showing genetically altered gene expression for SCZ, CD or UC compared to healthy controls. PPP3CA encodes the catalytic subunit calcineurin A; FKBP1A (also known as FKBP12) encodes a cis-trans prolyl isomerase that binds the immunosuppressant FK506 (tacrolimus). (b) The calcineurin inhibitor tacrolimus, used against various inflammatory diseases and as an immunosuppressant in organ-transplanted PSC-IBD patients, prevents NFAT signaling by binding to FKBP1A and causes inhibition of calcineurin. The reduced genetic expression of PPP3CA that we identified in intestinal and brain tissues causes an increased risk of SCZ and CD (Table 2).

\section{Supplementary Files}

This is a list of supplementary files associated with this preprint. Click to download.

- SupplTable1.xlsx

- SupplTable2.xlsx

- SupplTable3.xlsx

- SupplTable4.xIsx

- SupplTable5.xIsx

- SupplTable7.xlsx

- SupplTable6.xlsx

- SupplTable8.xIsx

- SupplTable9.xIsx

- SupplTable10.xIsx

- SupplTable11.xIsx

- SupplTable12.xIsx

- SupplTable13.xIsx

- SupplTable14.xIsx

- SupplementaryFile1.pdf 